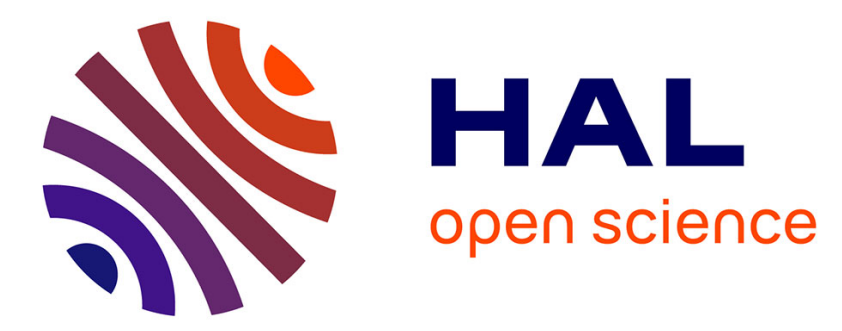

\title{
Calculation of Higher-Order Fluxes in Symmetric Cores-I: Theory
}

\author{
Jean Tommasi, Maxence Maillot, G. Rimpault
}

\section{To cite this version:}

Jean Tommasi, Maxence Maillot, G. Rimpault. Calculation of Higher-Order Fluxes in Symmetric Cores-I: Theory. Nuclear Science and Engineering, 2017, 184 (2), pp.174-189. 10.13182/NSE16-4 . cea-02387094

\section{HAL Id: cea-02387094 https://hal-cea.archives-ouvertes.fr/cea-02387094}

Submitted on 6 Jan 2020

HAL is a multi-disciplinary open access archive for the deposit and dissemination of scientific research documents, whether they are published or not. The documents may come from teaching and research institutions in France or abroad, or from public or private research centers.
L'archive ouverte pluridisciplinaire HAL, est destinée au dépôt et à la diffusion de documents scientifiques de niveau recherche, publiés ou non, émanant des établissements d'enseignement et de recherche français ou étrangers, des laboratoires publics ou privés. 


\section{Calculation of Higher-Order Fluxes in Symmetric Cores-I: Theory}

\section{Jean Tommasi, Maxence Maillot \& Gérald Rimpault}

To cite this article: Jean Tommasi, Maxence Maillot \& Gérald Rimpault (2016) Calculation of Higher-Order Fluxes in Symmetric Cores-I: Theory, Nuclear Science and Engineering, 184:2, 174-189, DOI: $10.13182 / N S E 16-4$

To link to this article: https://doi.org/10.13182/NSE16-4

\section{Published online: 27 Mar 2017.}

Submit your article to this journal ¿

Џ Article views: 25

Q View related articles $\asymp$

View Crossmark data ¿ 


\title{
Calculation of Higher-Order Fluxes in Symmetric Cores-I: Theory
}

\author{
Jean Tommasi,* Maxence Maillot, and Gérald Rimpault
}

CEA, DEN, SPRC, CEA-Cadarache, 13108 Saint Paul lez Durance, France

Received January 8, 2016

Accepted for Publication May 11, 2016

http://dx.doi.org/10.13182/NSE16-4

\begin{abstract}
In neutron chain systems with material symmetries, various k-eigenvalues of the neutron balance equation beyond the dominant one may be degenerate. Eigenfunctions can be partitioned into several classes according to their invariance properties with respect to the symmetry operations (mirror symmetries and rotations) keeping the material distribution in the system unchanged. Their calculation can be limited to a fraction of the system (sector) provided that innovative boundary conditions matching the symmetry classes are used, and whole-system eigenfunctions can then be unfolded from the solutions obtained over the sector. With power iteration as the method for searching $k$-eigenvalues, this use of the material symmetries to split the global problem into a variety of smaller-sized problems has several computational advantages: lower computation times and memory requirements, increased dominance ratios, lowered possible degeneracies in each subproblem, and possible parallel (separated) treatment of the subproblems. The implementation is discussed in a companion paper using diffusion and transport theories.
\end{abstract}

Keywords — Flux eigenmodes, symmetry, degeneracy.

Note - Some figures may be in color only in the electronic version.

\section{INTRODUCTION}

\section{I.A. General Background}

The $k$-eigenvalue neutron balance equation is written as

$$
\mathbf{A} \Phi=\frac{1}{k} \mathbf{F} \Phi
$$

where

$$
\begin{aligned}
\Phi(\vec{r}, E, \vec{\Omega}, t)= & \text { neutron flux density at a given posi- } \\
& \text { tion, energy, and direction } \\
\mathbf{F}= & \text { operator modeling neutron produc- } \\
& \text { tion by fission } \\
\mathbf{A}= & \text { operator collecting contributions } \\
& \text { of streaming (leaking) neutrons }(\mathbf{L}), \\
& \text { neutrons entering a collision }(\mathbf{C}) \text {, and } \\
& \text { neutrons emerging from a collision }(\mathbf{S}) .
\end{aligned}
$$

*E-mail: jean.tommasi@cea.fr
With standard notation and variables omitted when not explicitly necessary,

$$
\mathbf{F} \Phi=\frac{1}{4 \pi} \iint \chi\left(E^{\prime} \rightarrow E\right) \nu \Sigma_{f}\left(E^{\prime}\right) \Phi\left(E^{\prime}, \vec{\Omega}^{\prime}\right) d E^{\prime} d^{2} \Omega^{\prime}
$$

and

$$
\begin{aligned}
\mathbf{A} \Phi & =\vec{\Omega} \cdot \vec{\nabla} \Phi+\Sigma_{t} \Phi-\iint \Sigma_{s}\left(E^{\prime} \rightarrow E, \overrightarrow{\Omega^{\prime}} \rightarrow \vec{\Omega}\right) \\
& \times \Phi\left(E^{\prime}, \vec{\Omega}^{\prime}\right) d E^{\prime} d^{2} \Omega^{\prime}=\mathbf{L} \Phi+\mathbf{C} \Phi-\mathbf{S} \Phi
\end{aligned}
$$

A dominant, simple, and positive $k$-eigenvalue always exists and is called the fundamental (fission) multiplication factor $k_{0}$, associated with a positive fundamental flux $\Phi_{0}$. All other $k$-eigenfunctions are called higher modes or harmonics. The $k$-spectrum is generally deemed to be discrete and real, even if it this seems to be proved only under restrictive conditions (e.g., monoenergetic transport). If not theoretically, this 
assumption is nevertheless supported by numerical evidence on real-life problems. ${ }^{1}$ In the following, we will make this assumption.

The ratio $k_{1} / k_{0}$ of the second largest $k$-eigenvalue to the fundamental one is called the dominance ratio and is used as a measure of the speed of convergence of the power iteration method used to get $\left(k_{0}, \Phi_{0}\right)$ in deterministic or Monte Carlo solvers. In another form called the eigenvalue separation EVS $=k_{0} k_{1} /\left(k_{0}-k_{1}\right)$, the second largest $k$-eigenvalue is used as a measure of core decoupling and sensitivity to local perturbations. ${ }^{2-4}$ The EVS is not a pure calculation parameter, but is, at least indirectly, accessible to experiment. ${ }^{5-11}$ A larger number of $k$-eigenvalues and eigenfunctions can be used for the modal decomposition of the fundamental flux of a perturbed situation using the eigenfunctions associated with a reference situation, ${ }^{12}$ for the prompt decay constant or exponential experiment analysis, ${ }^{13}$ and for the stability analysis for a boiling water reactor. ${ }^{14,15}$ However, theoretically, this set of $k$-eigenfunctions is not complete; furthermore, when the perturbation is localized in space or shifts the neutron spectrum, a rapidly growing number of higher modes is needed to reach a given precision, if this does not prove unachievable at all. Nevertheless, practical decompositions, i.e., over a limited number of eigenfunctions, may prove meaningful, depending on the problem considered.

\section{I.B. Orthogonality Properties of $k$-Eigenfunctions}

The inner product of two possibly complex-valued functions is defined as the hermitic form:

$$
\left\langle\Phi_{1}, \Phi_{2}\right\rangle=\int \bar{\Phi}_{1}(\vec{r}, E, \vec{\Omega}) \Phi_{2}(\vec{r}, E, \vec{\Omega}) d^{3} r d E d^{2} \Omega
$$

(complex functions will be used in the following as mathematical intermediaries, even if $k$-eigenfunctions are real). The adjoint $\mathbf{P}^{+}$of a given operator $\mathbf{P}$ is defined as obeying the relation $\left\langle\Phi_{1}, \mathbf{P} \Phi_{2}\right\rangle=\left\langle\mathbf{P}^{+} \Phi_{1}, \Phi_{2}\right\rangle$ for any $\Phi_{1}$ and $\Phi_{2}$. For the operators involved in the neutron balance equation, with appropriate boundary conditions, adjoint operators are obtained reverting all kernels and changing all derivatives to their opposites:

$$
\mathbf{F}^{+} \Psi=\frac{1}{4 \pi} \iint \chi\left(E \rightarrow E^{\prime}\right) \nu \Sigma_{f}(E) \Psi\left(E^{\prime}, \vec{\Omega}^{\prime}, t\right) d E^{\prime} d^{2} \Omega^{\prime}
$$

and

$$
\begin{gathered}
\mathbf{A}^{+} \Psi=-\vec{\Omega} \cdot \vec{\nabla} \Psi+\Sigma_{t} \Psi+\iint \Sigma_{s}\left(E \rightarrow E^{\prime}, \vec{\Omega} \rightarrow \vec{\Omega}^{\prime}\right) \\
\times \Psi\left(E^{\prime}, \vec{\Omega}^{\prime}\right) d E^{\prime} d^{2} \Omega^{\prime}
\end{gathered}
$$

so that the forward and adjoint balance equations over a convex volume $V$, in integrodifferential transport form, with void boundary conditions, can be written as

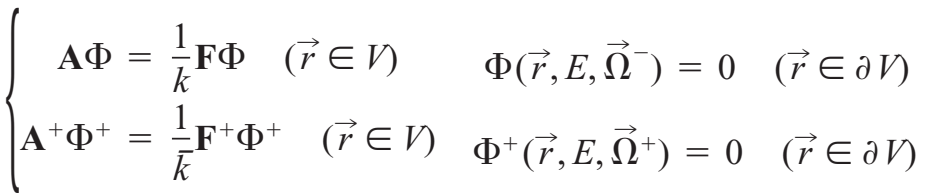

(on boundary $\partial V$ of volume $V, \vec{\Omega}^{-}$stands for any inward-pointing direction and $\vec{\Omega}^{+}$for any outward-pointing direction). The $k$-eigenvalues being assumed all real, then the forward and adjoint $k$-eigenvalue equations share the same eigenvalue spectrum.

It can be easily checked, by taking the inner product of the first equation by $\Phi^{\prime}$ and of the second by $\Phi^{+}$and subtracting, that if $\mathbf{A}^{+} \Phi^{+}=\frac{1}{k} \mathbf{F}^{+} \Phi^{+}$and $\mathbf{A} \Phi^{\prime}=\frac{1}{k^{\prime}} \mathbf{F} \Phi^{\prime}$ then $\left(k-k^{\prime}\right)\left\langle\Phi^{+}, \mathbf{F} \Phi^{\prime}\right\rangle=0$. Consequently, the adjoint and forward eigenfunctions corresponding to two distinct eigenvalues are $\mathbf{F}$-orthogonal, i.e., $\left\langle\Phi^{+}, \mathbf{F} \Phi^{\prime}\right\rangle=0$ if $k \neq k^{\prime}$.

\section{I.C. Numerical Computation Methods for $k$-Eigenvalues and Eigenfunctions}

For its conceptual simplicity, and as the dominant $k$-eigenvalue is simple, the power iteration method has been widely used, both in deterministic and Monte Carlo codes to access the fundamental eigenpair $\left(k_{0}, \Phi_{0}\right)$. A sketch of this iterative process is

$$
\left\{\begin{aligned}
(\mathbf{L}+\mathbf{C}) \Phi_{0}^{(q+1)} & =\mathbf{S} \Phi_{0}^{(q)}+\frac{1}{k_{0}^{(q)}} \mathbf{F} \Phi_{0}^{(q)} \\
k_{0}^{(q+1)} & =\frac{\left\|\mathbf{F} \Phi_{0}^{(q+1)}\right\|}{\left\|\mathbf{A} \Phi_{0}^{(q+1)}\right\|},
\end{aligned}\right.
$$


where $q$ is the outer iteration index; $\Phi_{0}^{(q+1)}$ in the first equation is obtained, using numerical linear algebra techniques, through an iterative process called inner iterations. This iterative process converges toward the first simple eigenpair $\left(k_{0}, \Phi_{0}\right)$. The speed of convergence is known to be dictated by the dominance ratio $k_{1} / k_{0}$ of the first harmonic eigenvalue to the fundamental one. If the dominance ratio approaches unity, as occurs, for example, with large loosely coupled systems, the convergence of the power iteration methods gets very slow and acceleration techniques are required, such as Chebyshev or diffusion synthetic accelerations. ${ }^{16}$

The power iteration method can be extended to access higher-order eigenfunctions and eigenvalues, taking advantage of the $\mathbf{F}$-orthogonality properties of eigenfunctions associated with distinct eigenvalues for cleaning the output of the inner iterations from all previously calculated eigenfunctions; this is called the filtering technique and it can be sketched as follows for the forward harmonic number $h$ :

$$
\left\{\begin{array}{l}
(\mathbf{L}+\mathbf{C}) \tilde{\Phi}_{h}^{(q+1)}=\mathbf{S} \Phi_{h}^{(q)}+\frac{1}{k_{h}^{(q)}} \mathbf{F} \Phi_{h}^{(q)} \\
\Phi_{h}^{(q+1)}=\tilde{\Phi}_{h}^{(q+1)}-\sum_{i=0}^{h-1} \frac{\left\langle\Phi_{i}^{+}, \mathbf{F} \tilde{\Phi}_{h}^{(q+1)}\right\rangle}{\left\langle\Phi_{i}^{+}, \mathbf{F} \Phi_{i}\right\rangle} \Phi_{i} \\
k_{h}^{(q+1)}=\frac{\left\|\mathbf{F} \Phi_{h}^{(q+1)}\right\|}{\left\|\mathbf{A} \Phi_{h}^{(q+1)}\right\|} .
\end{array}\right.
$$

If all the eigenvalues are simple, this method works smoothly. If there are multiple (degenerate) $k$-eigenvalues, i.e., if the successive $k$-eigenspace is spanned by $p>1$ linearly independent eigenvectors, the method still converges toward the eigenvalue of the successive dominant eigenspace; however, convergence to an eigenvector is not ensured, as drifts within the $p$-dimensional eigenspace may occur during the iterations, preventing them from reaching a point-by-point flux convergence. Nevertheless, once a tight eigenvalue convergence is reached, the flux is practically an eigenfunction within the $p$-dimensional eigenspace. Then, the orthogonal filtering technique can be used to successively span $p$ independent directions of the eigenspace. (All the previous statements hold out of exceptional cases.) Irreducible convergence problems may occur only with distinct eigenvalues sharing the same modulus. If we assume all eigenvalues to be real and positive, this cannot occur.

The power iteration method with the filtering technique to compute higher eigenpairs has been extensively used in deterministic codes and has been extended recently to Monte Carlo codes. ${ }^{17-23}$ However, other methods may prove quicker, more efficient, or more easily parallelizable than the power method, for example, subspace iteration methods such as Krylov or Krylov-Arnoldi methods for deterministic calculations ${ }^{14,24,25}$ or fission matrix methods for both deterministic ${ }^{26}$ and Monte Carlo calculations. ${ }^{1,27}$ These methods give simultaneous access to estimates of several eigenvalues and eigenfunctions.

\section{I.D. Material Symmetries, Eigenvalue Degeneracies, and Problem Size Reduction}

If there are material symmetries in the system, some eigenvalues may be degenerate. Multiplicity of degenerate eigenvalues can be linked to symmetry invariances of the reactor material distribution, e.g., through group theory considerations. ${ }^{28,29}$ Moreover, material symmetries can be exploited to solve the neutron balance equation on reducedsize geometries with appropriate innovative boundary conditions. As an example for mirror symmetries, implicit ${ }^{28}$ or explicit $^{30}$ use can be made of antisymmetric reflective boundary conditions in addition to the standard, symmetric, reflective boundary condition.

In a more radical way, and to avoid possible convergence problems, all existing symmetries can be broken using small localized perturbations of the material distribution within the reactor and then the $k$-eigenfunctions of the asymmetric perturbed system may be used as an expansion basis to approximate the eigenfunctions of the symmetric system. ${ }^{31}$

Real-life reactors, once burnup occurs or control rods are inserted at different heights, no longer exhibit global material arrangement symmetries, but start-of-life designed industrial reactor cores most often do. Moreover, critical experiments in zero-power reactors are often, at least in a first approximation, designed as presenting material symmetries. The point developed hereafter is that besides the more recent methods hinted at (subspace iteration and fission matrix), the old power iteration method can be amended to compute efficiently the higher modes of symmetric systems. Advantage can be taken of material symmetries to split the computational task into several classes of smaller-sized problems subject to specific boundary conditions, not only for mirror symmetries but also for rotation symmetries. This results in several positive features:

1. Each class of solutions is worked out separately (and possibly on a dedicated processing unit) on a fraction of the global system, meaning less memory and a lower computational burden.

2 . The whole $k$-spectrum is partitioned into the various classes, increasing the dominance ratios in each class with respect to the whole-core problem. 
3. The degeneracy of any given $k$-eigenvalue is also partitioned into the symmetry classes (i.e., a degenerate $k$-eigenvalue for the whole core may be simple in a given symmetry class).

\section{I.E. Outline}

The symmetry invariance is formalized in Sec. II, from the material distribution in the system to functions such as the neutron flux, and operators acting on such functions. Section III recalls a few results from group representation theory justifying the approach used in the following sections. Section IV is devoted to the individuation of the symmetry classes corresponding to order- $n$ rotation symmetry, and the associated innovative boundary conditions, which, to our knowledge, do not seem to have been used in finite difference neutron diffusion or transport codes. Mirror symmetries are addressed in Sec. V, and the results obtained are combined in Sec. VI when the symmetry group of the system contains at the same time mirror symmetries and rotations with complex-valued eigenvalues, i.e., in practical cases, rotations of order 3, 4, or 6.

In Sec. VII, a simple analytic monoenergetic diffusion example (eigenvalues and eigenfunctions of the Laplacian in the equilateral triangle) is given to support and illustrate the theoretical results on symmetry classes and associated eigenfunction shapes; additionally, the example of eigenfunctions of the Laplacian in the regular hexagon is sketched to show how the classifications associated with rotation or mirror symmetry invariances match together.

The implementation in an existing code package, verification, and applications are worked out in a companion paper. ${ }^{32}$

\section{SYMMETRY-INVARIANT FUNCTIONS AND OPERATORS}

\section{II.A. Symmetry Operations}

The material distribution in the neutron chain reactor (system) is assumed invariant by the application of symmetry operations belonging to a group $G$. A symmetry operation is a one-to-one point transform $S$ preserving distances (isometric), i.e., such that $d[S(A), S(B)]=d(A, B)$ for any two points $A$ and $B$. The material distribution is $G$-invariant if for any nuclide distribution $N$ and any $S$ in $G$ we have $N(\vec{r})=N[S(\vec{r})]$ at any position.

In the Euclidean vector space, for every symmetry operation $S$ there corresponds an orthogonal matrix $\mathbf{M}_{S}$. A symmetry operation $S$ is either direct, if $\operatorname{det}\left(\mathbf{M}_{S}\right)=+1$, or inverse, if $\operatorname{det}\left(\mathbf{M}_{S}\right)=-1$. For systems of finite spatial extension, translations are not allowed as symmetry operations and direct symmetries are rotations around an axis; inverse symmetries are either mirror symmetries with respect to a plane or products of such mirror symmetries with rotations. If it exists, the lowest positive integer, such that $S^{n}=E$ ( $E$ standing for the identity), will be called the order of the discrete symmetry operation $S$. A mirror symmetry $S$ is involutive, i.e., $S^{2}=E$. Only finite-order rotations will be dealt with here, i.e., rotations whose angle is $2 \pi / n$, with integer $n$. Then, $G$ is a finite group.

\section{II.B. Symmetry-Invariant Functions}

For any symmetry operation $S \in G$ in three-dimensional Euclidean space, we associate a symmetry operation $\mathbf{S}$ in the algebra of scalar functions $\Phi$ of $\omega=(\vec{r}, E, \vec{\Omega})$ by the condition $\Phi(\omega)=(\mathbf{S} \Phi)[S(\omega)]$ : in words, operating the transformed function $\mathbf{S} \Phi$ on the transformed point $S(\omega)$ yields the same result as operating the initial function $\Phi$ on the initial point $\omega$. Formally, $\mathbf{S}$ is defined by

$$
\begin{aligned}
\text { S: } \Phi(\omega) & \mapsto(\mathbf{S} \Phi)(\omega)=\Phi\left[S^{-1}(\omega)\right] \\
= & \Phi\left[S^{-1}(\vec{r}), E, S^{-1}(\vec{\Omega})\right] .
\end{aligned}
$$

A function $\Phi$ is said to be $\mathbf{S}$-invariant if $\mathbf{S} \Phi=\Phi$ and $\mathbf{S}$-antisymmetric if $\mathbf{S} \Phi=-\Phi$. It is easily checked that $\mathbf{S}$ is invertible: if $\mathbf{S}$ is associated with $S$, then $\mathbf{S}^{-1}$ is associated with $S^{-1}$, and then the symmetry operation $\mathbf{S}$ maps the function space algebra onto itself, with the following relations:

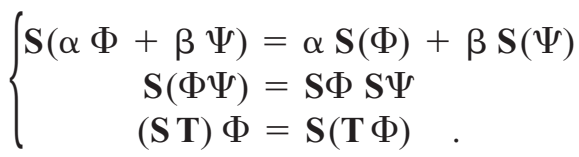

Any spatial symmetry $S$, being an orthogonal transform, maps any portion of the unit sphere onto an equivalent portion (i.e., of same area), and any spatial domain onto an equivalent domain (i.e., of same volume). Hence, the absolute value of the Jacobian of $S$ is 1 . Using the already defined inner product, we have then

$$
\begin{aligned}
\langle\Psi, \mathbf{S} \Phi\rangle & =\int \bar{\Psi}(\omega) \Phi\left[S^{-1}(\omega)\right] d \omega \\
& =\int \bar{\Psi}\left[S\left(\omega^{\prime}\right)\right] \Phi\left(\omega^{\prime}\right) d \omega^{\prime}=\left\langle\mathbf{S}^{-1} \Psi, \Phi\right\rangle,
\end{aligned}
$$

meaning that $\mathbf{S}$ is unitary $\left(\mathbf{S}^{+}=\mathbf{S}^{-1}\right)$, and that the inner product is $\mathbf{S}$-invariant,

$$
\langle\mathbf{S} \Psi, \mathbf{S} \Phi\rangle=\langle\Psi, \Phi\rangle .
$$


It follows that all eigenvalues of the operator $\mathbf{S}$ are of unit modulus. Then, if $\lambda$ and $\mu$ are eigenvalues of $\mathbf{S}$ for, respectively, eigenfunctions $\Phi$ and $\Psi$,

$$
\begin{aligned}
\langle\Phi, \Psi\rangle & =\langle\mathbf{S} \Phi, \mathbf{S} \Psi\rangle=\langle\lambda \Phi, \mu \Psi\rangle \\
& =\bar{\lambda} \mu\langle\Phi, \Psi\rangle=\frac{\mu}{\lambda}\langle\Phi, \Psi\rangle
\end{aligned}
$$

So, eigenfunctions of $\mathbf{S}$ corresponding to distinct eigenvalues are orthogonal. The order of $S$ is also the order of $\mathbf{S}$, and if $S$ is of order $n$, any eigenvalue $\lambda$ of $\mathbf{S}$ must obey the condition $\lambda^{n}=1$. Mirror symmetries being involutive, their only possible eigenvalues are \pm 1 , whereas the eigenvalues of an order- $n$ rotation are the $n$ roots of unity, i.e., the $\varepsilon^{m}=c_{m}+i s_{m}(m=0, \ldots, n-1)$, using the notations

$$
\varepsilon=\exp \left(\frac{2 i \pi}{n}\right), c_{m}=\cos \left(m \frac{2 \pi}{n}\right), s_{m}=\sin \left(m \frac{2 \pi}{n}\right) .
$$

The only possible real eigenvalues for discrete rotations are $\varepsilon^{0}=1$, in every case, and $\varepsilon^{n / 2}=-1$ if $n$ is even. The presence of possible complex R-eigenpairs is the reason why complex functions are accepted and the inner product used is hermitic.

\section{II.C. Symmetry-Invariant Operators and Consequences for $k$-Eigenfunctions}

An operator $\mathbf{P}$ is said to be invariant for the symmetry operation $\mathbf{S}$ (or $\mathbf{S}$-invariant) if the operation of $\mathbf{P}$ on any function $\Phi$ matches its application to the transformed function $\mathbf{S} \Phi$, according to Fig. 1 . This means that $\mathbf{S}$ and $\mathbf{P}$ commute. As the symmetry operation $S$ accounts for mapping any orthonormal frame onto another orthonormal frame, this occurs if $\mathbf{P}$ takes the same form in the initial orthonormal frame and its transform by $S$. This is the case for the operators $\mathbf{A}$ and $\mathbf{F}$ used in the neutron balance equation if they are constructed on $S$-invariant material distributions, in particular because scattering cross sections depend only on the $S$-invariant inner product $\vec{\Omega} \cdot \vec{\Omega}^{\prime}$ between the directions of neutrons entering and exiting the scattering reaction. Consequently,

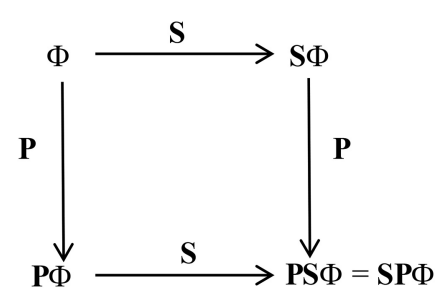

Fig. 1. Visualization of the condition for operator $\mathbf{P}$ to be invariant under symmetry operation $\mathbf{S}$.

$$
\begin{gathered}
\left(\mathbf{A}-\frac{\mathbf{F}}{k}\right) \Phi=0 \Rightarrow \mathbf{S}\left(\mathbf{A}-\frac{\mathbf{F}}{k}\right) \Phi=0 \\
\Rightarrow\left(\mathbf{A}-\frac{\mathbf{F}}{k}\right)(\mathbf{S} \Phi)=0 .
\end{gathered}
$$

Intuitively, if the material distribution in the system is invariant under the symmetry operations of a group $G$, and if $\Phi$ is a $k$-eigenfunction of the system, then for any $S$ in $G$ the transformed neutron flux $\mathbf{S} \Phi$ is also a $k$-eigenfunction of the system, for the same k-eigenvalue.

\section{RESULTS OF GROUP REPRESENTATION THEORY}

We briefly recall a few results of finite group representation theory, as they justify the way we will proceed in the following sections; more details of the vocabulary and demonstrations can be found in any textbook on the subject. Given a finite group $G$, a $p$-dimensional representation $r$ of $G$ associates with any element $g$ in $G$ a $p \times p$ complex matrix $r(g)$ and is such that for any two elements $g$ and $g^{\prime}$ in $G$, $r\left(g g^{\prime}\right)=r(g) r\left(g^{\prime}\right)$. Such a representation $r$ is reducible if, using a suitable change of basis vectors, all the $p \times p$ matrices of the representation can be cast simultaneously into equivalent block-diagonal forms with diagonal blocks of size $<p$. A result of group representation theory is that, for any finite group $G$, there exists only a finite number $N_{G}$ of nonequivalent irreducible representations, which are the building blocks for any representation of this group.

Given a group $G=\left\{S_{1}, S_{2}, \ldots, S_{n}\right\}$ of order $n$, and a $n$-dimensional vector space $T$ with basis $\left(e_{1}, e_{2}, \ldots, e_{n}\right)$, the regular representation $\rho$ of $G$ is defined as the representation by $n \times n$ matrices $\mathbf{M}_{i}=\rho\left(S_{i}\right)(i=1, \ldots, n)$ operating on the basis vectors as $\mathbf{M}_{i}\left(e_{j}\right)=e_{k}$ if $S_{i} S_{j}=$ $S_{k}$. It is easy to check that $\rho\left(S_{i} S_{j}\right)=\rho\left(S_{i}\right) \rho\left(S_{j}\right)$, as it is required for $\rho$ to be a representation. This regular representation is reducible as a direct sum over the irreducible representations of $G$, each irreducible representation being counted with a multiplicity equal to its dimension [in particular, this means that $\left.\sum_{i=1}^{N_{G}}\left(\operatorname{dim} \rho_{1}\right)^{2}=n\right]$. As for any representation, it can be projected onto its irreducible component, and reconstructed back from its projections:

1. $\quad \mathbf{P}_{i}=\frac{\operatorname{dim} \rho_{i}}{|G|} \sum_{g \in G} \overline{\chi_{i}(g)} \rho(g)$ is the projection operator on irreducible component number $i$.

2. $\sum_{i=1}^{N_{G}} \mathbf{P}_{i}=\rho(e)=\mathbf{E}$ ( $e$ is the unit element of $G$, and $\mathbf{E}$ is the $n \times n$ identity matrix).

The dimensions $\operatorname{dim} \rho_{i}$ of the irreducible components and their characters, i.e., the complex numbers $\chi_{i}(g)$ asso- 
ciated with each element of the group, can be found in the character tables given in textbooks.

Additionally, all irreducible representations of a commutative group are of dimension 1: then $\rho_{i}(g)$ is a $1 \times 1$ complex matrix, assimilated to the complex number $\chi_{i}(g)$, so that $\rho_{i}\left(g^{\prime}\right) \mathbf{P}_{i}=\chi_{i}\left(g^{\prime}\right) \mathbf{P}_{i}$. In this case, the regular representation $\rho$ contains all the one-dimensional irreducible representations once.

We will use these results as follows. If $G$ is a commutative group of symmetry operations acting on functions according to Eq. (1) then, for any function $f$, $\mathbf{P}_{i} f=\frac{1}{|G|} \sum_{\mathbf{S} \in G} \overline{\chi_{i}(\mathbf{S})} \mathbf{S} f$ is an eigenvector for each symmetry operation $\mathbf{S}^{\prime}$ in $G$, the eigenvalue being equal to the character of $\mathbf{S}^{\prime}$ in the irreducible representation $\rho_{i}$ : $\mathbf{S}^{\prime}\left(\mathbf{P}_{i} f\right)=\chi_{i}\left(\mathbf{S}^{\prime}\right) \mathbf{P}_{i} f$. Together with the relation $\sum_{i=1}^{N_{G}} \mathbf{P}_{i} f=f$, this shows that, given a symmetry operation $\mathbf{S}$, any function $f$ can be expanded into a sum of eigenfunctions of $\mathbf{S}$. Going back to the initial problem, if the material distribution is invariant under the action of a commutative group $G$ of symmetry operations, the search for solutions of the balance equation $\left(\mathbf{A}-\frac{\mathbf{F}}{k}\right) \Phi=0$ can be split into as many subproblems as there are irreducible representations of $G$, with the additional conditions that $\Phi$ is also an eigenfunction of symmetry operations, with their characters as eigenvalues. As will be shown below, these additional conditions make it possible to work on only a part of the whole system, using appropriate and simple boundary conditions.

\section{INVARIANCE UNDER DISCRETE ROTATIONS}

We assume the system is of prismatic shape, as are many industrial reactors, with a discrete rotation axis being taken as the vertical direction. A system invariant under a discrete rotation $\mathbf{R}$ of order $n$, i.e., such that $\mathbf{R}^{n}=\mathbf{E}$, is invariant under the action of the commutative cyclic group of order $n, C_{n}=\left\{\mathbf{E}, \mathbf{R}, \mathbf{R}^{2}, \ldots, \mathbf{R}^{n-1}\right\}$.

\section{IV.A. Problem Decomposition}

According to Sec. III and the character table of $C_{n}$, the operators projecting any complex-valued function $Z$ onto the mutually orthogonal $\mathbf{R}$-invariant subspaces are

$$
\mathbf{P}_{m} Z=\frac{1}{n} \sum_{p=0}^{n-1} \varepsilon^{-p m} \mathbf{R}^{p} Z(0 \leq m \leq n-1) .
$$

In addition, $\mathbf{P}_{m} Z$ is an eigenvector of rotation operator $\mathbf{R}$ for eigenvalue $\varepsilon^{m}$. We define the real and imaginary parts of $\mathbf{P}_{m}$ by setting $\mathbf{P}_{m}=\mathbf{u}_{m}+i \mathbf{v}_{m}$, with

$$
\mathbf{u}_{m} Z=\frac{1}{n} \sum_{p=0}^{n-1} c_{-p m} \mathbf{R}^{p} Z \text { and } \mathbf{v}_{m} Z=\frac{1}{n} \sum_{p=0}^{n-1} s_{-p m} \mathbf{R}^{p} Z .
$$

As seen in Sec. III, any function $Z$ is the sum of its projections onto the R-invariant subspaces: $Z=\sum_{m=0}^{n-1} \mathbf{P}_{m} Z$.

We also define the conjugate of $\mathbf{P}_{m}$ as $\overline{\mathbf{P}}_{m} \stackrel{m=0}{=} \mathbf{u}_{m}-i \mathbf{v}_{m}$, i.e., $\overline{\mathbf{P}}_{m} f=\frac{1}{n} \sum_{p=0}^{n-1} \varepsilon^{+p m} \mathbf{R}^{p} f$ for any function $f$. Then $\mathbf{R} \overline{\mathbf{P}}_{m} f=\varepsilon^{-m} \overline{\mathbf{P}}_{m} f$. If $\mathbf{v}_{m} \neq 0$ and $\varepsilon^{m} \neq \varepsilon^{-m}$, then as the intersection of the orthogonal R-eigenspaces corresponding to two distinct eigenvalues reduces to the null vector, $\overline{\mathbf{P}}_{m} \mathbf{P}_{m}=\mathbf{P}_{m} \overline{\mathbf{P}}_{m}=0$.

Then, by developing in $\mathbf{u}_{m}$ and $\mathbf{v}_{m}$,

$$
\mathbf{P}_{m}^{2}=\mathbf{P}_{m} \rightarrow\left\{\begin{array}{l}
\mathbf{u}_{m}^{2}-\mathbf{v}_{m}^{2}=\mathbf{u}_{m} \\
\mathbf{u}_{m} \mathbf{v}_{m}+\mathbf{v}_{m} \mathbf{u}_{m}=\mathbf{v}_{m}
\end{array}\right.
$$

and

$$
\overline{\mathbf{P}}_{m} \mathbf{P}_{m}=0 \rightarrow\left\{\begin{array}{l}
\mathbf{u}_{m}^{2}+\mathbf{v}_{m}^{2}=0 \\
\mathbf{u}_{m} \mathbf{v}_{m}-\mathbf{v}_{m} \mathbf{u}_{m}=0
\end{array}\right.
$$

Consequently,

$$
\mathbf{u}_{m}^{2}=\frac{\mathbf{u}_{m}}{2} ; \mathbf{v}_{m}^{2}=-\frac{\mathbf{u}_{m}}{2} ; \mathbf{u}_{m} \mathbf{v}_{m}=\mathbf{v}_{m} \mathbf{u}_{m}=\frac{\mathbf{v}_{m}}{2}\left(\text { if } \mathbf{v}_{m} \neq 0\right)
$$

If we write a complex-valued function $Z$ of the $\mathbf{R}$-invariant subspace associated with eigenvalue $\varepsilon^{m}$ as the sum of its real and imaginary parts, $Z=U+i V$, the relation $\mathbf{R} Z=\varepsilon^{m} Z$ may be written as $\mathbf{R}(U+i V)=$ $\left(c_{m}+i s_{m}\right)(U+i V)$, and so

$$
\left\{\begin{array}{l}
\mathbf{R} U=c_{m} U-s_{m} V \\
\mathbf{R} V=s_{m} U+c_{m} V .
\end{array}\right.
$$

If $s_{m}=0$, we can deal with real functions only. This occurs for $m=0$, and involves then $\mathbf{R}$-invariant solutions $(\mathbf{R} U=U)$. When $n$ is even, this occurs also for $m=n / 2$, and involves R-antisymmetric solutions $(\mathbf{R} U=-U)$. For any other value of $m, U$ and $V$ span a plane globally invariant by $\mathbf{R}$ and we call this a coupled problem. We can restrict these coupled problems to $0<m<n / 2$, as Eq. (6) shows that if $(U, V)$ is a solution of the coupled problem for $\mathbf{R}$-eigenvalue $\varepsilon^{m}$, then $(V, U)$ is a solution of the coupled problem for eigenvalue $\varepsilon^{n-m}$. As a consequence of Eq. $\left(10^{\prime \prime}\right)$ demonstrated in the next section, $U$ and $V$ are linearly independent, with $\langle U, U\rangle=\langle V, V\rangle$ and $\langle U, V\rangle=0$. Furthermore, the characteristic polynomial associated with the $2 \times 2$ matrix $\left(\begin{array}{ll}c_{m} & s_{m} \\ s_{m} & c_{m}\end{array}\right)$ shows that

$$
U-2 c_{m} \mathbf{R} U+\mathbf{R}^{2} U=V-2 c_{m} \mathbf{R} V+\mathbf{R}^{2} V=0 .
$$


To sum up, for a system invariant by rotation $R$ of finite order $n$, the search for nonnull real eigenfunctions of the neutron balance equation can be partitioned into distinct subproblems:

1. the search for R-invariant functions (such that $\mathbf{R} \Phi=\Phi)$

2. only if $n$ is even: the search for $\mathbf{R}$-antisymmetric functions (such that $\mathbf{R} \Phi=-\Phi$ )

3. for $0<m<n / 2$ : the search for real function couples $(U, V)$ such that

$$
\left\{\begin{array}{l}
\mathbf{R} U=c_{m} U-s_{m} V \\
\mathbf{R} V=s_{m} U+c_{m} V
\end{array} .\right.
$$

\section{IV.B. Reduced-Sized Problems and Unfolding}

We shall proceed to show that the distinct subproblems can be solved on a limited spatial domain $D$ (called a sector) representing a fraction $1 / n$ of the system, with appropriate boundary conditions, and that the solutions obtained on this sector can be unfolded to obtain the flux distributions and associated integral values over the whole system.

$D$ is a sector containing the rotation axis and extending over an angle $2 \pi / n$, with boundary $\partial D=$ $\partial D^{-} \cup \partial D^{+} \cup \partial D^{e x t}$. $\partial D^{e x t}$ is the external boundary, where the boundary condition of the whole-system equation holds. $\partial D^{-}$and $\partial D^{+}=R\left(\partial D^{-}\right)$are the left and right edges of the sector, between which generic phase space elements can be paired as

$$
\left\{\begin{array}{l}
\omega^{-}=(\vec{r}, E, \vec{\Omega}) \\
\omega^{+}=R\left(\omega^{-}\right)=[R(\vec{r}), E, R(\vec{\Omega})]
\end{array}\left(\vec{r} \in \partial D^{-}\right) .\right.
$$

Assume $Z$ to be simultaneously an eigenfunction of the rotation operator $\mathbf{R}$ and a solution of the balance equation over the whole spatial domain: $\mathbf{R} Z=\varepsilon^{m} Z$ and $\left(\mathbf{A}-\frac{\mathbf{F}}{k}\right) Z=0$ with a void boundary condition. The restriction $z$ of $Z$ to sector $D$ is such that

$$
\begin{aligned}
& \left(\mathbf{A}-\frac{\mathbf{F}}{k}\right) z=0, \\
& z=0,
\end{aligned}
$$

Boundary condition of the global problem, $z\left(\omega^{-}\right)=\varepsilon^{m} z\left(\omega^{+}\right)$, on sector $D$;

outside sector $D$;

on $\partial D^{\text {ext }}$;

connecting $\partial D^{-}$to $\partial D^{+}$.

The last boundary condition results from the conjunction of

$$
\begin{aligned}
(\mathbf{R} Z)\left(\omega^{+}\right) & =Z\left[R^{-1}\left(\omega^{+}\right)\right]=Z\left(\omega^{-}\right) & & (\text {by definition}) ; \\
\mathbf{R} Z\left(\omega^{+}\right) & =\varepsilon^{m} Z\left(\omega^{+}\right) & & (Z \text { eigenfunction of } \mathbf{R}) .
\end{aligned}
$$

If $Z=U+i V, U$ and $V$ are real, and, correspondingly, $z=u+i v$, Table I details these sector boundary conditions according to the rotation class of the problem solved. The boundary condition for the $\mathbf{R}$-invariant problem is quite classical. To our knowledge, the boundary conditions for the R-antisymmetric problem and for the coupled problems do not seem to have been used in neutron transport or diffusion solvers. $Z$ (on the whole spatial domain) can be reconstructed from $z$ (nonnull only on sector $D$ ) as

$$
Z=\sum_{p=0}^{n-1} \varepsilon^{-p m} \mathbf{R}^{p}
$$

\begin{tabular}{|c|c|}
\hline Rotation Class & Boundary Condition Connecting $\partial D^{-}$to $\partial D^{+}=R\left(\partial D^{-}\right)$ \\
\hline $\begin{array}{l}\mathbf{R} \text {-invariant }(m=0, \mathbf{R} U=U) \\
\mathbf{R} \text {-antisymmetric }(n \text { even, } m=n / 2, \mathbf{R} U=-U) \\
\text { Coupled problems }\left(\varepsilon^{2 m} \neq 1, \mathbf{R} Z=\varepsilon^{m} Z \text {, i.e., }\left\{\begin{array}{l}\mathbf{R} U=c_{m} U-s_{m} V \\
\mathbf{R} V=s_{m} U+c_{m} V\end{array}\right)\right.\end{array}$ & $\begin{array}{l}u\left(\omega^{-}\right)=u\left(\omega^{+}\right) \\
u\left(\omega^{-}\right)=-u\left(\omega^{+}\right) \\
z\left(\omega^{-}\right)=\varepsilon^{m} z\left(\omega^{+}\right), \text {i.e., }\left\{\begin{array}{l}u\left(\omega^{-}\right)=c_{m} u\left(\omega^{+}\right)-s_{m} v\left(\omega^{+}\right) \\
v\left(\omega^{-}\right)=s_{m} u\left(\omega^{+}\right)+c_{m} v\left(\omega^{+}\right)\end{array}\right.\end{array}$ \\
\hline
\end{tabular}

It can be checked that $\mathbf{R} Z=\sum_{p=0}^{n-1} \varepsilon^{-p m} \mathbf{R}^{p+1} z=\varepsilon^{m} \sum_{p=0}^{n-1} \varepsilon^{-(p+1) m} \mathbf{R}^{p+1} z=\varepsilon^{m} Z$ and that the boundary conditions ensure continuity of $Z$ at sector edges.

TABLE I

Sector Boundary Conditions According to the Rotation Class 
Integrals such as $\left\langle Z, \mathbf{Q} Z^{\prime}\right\rangle$, where $Z$ and $Z^{\prime}$ are solutions of the balance equation that are eigenfunctions of $\mathbf{R}$ for the same eigenvalue and $\mathbf{Q}$ is any operator commuting with $\mathbf{R}$, can be expressed using integrals only involving the solutions $z$ and $z^{\prime}$ on sector $D$ :

$$
\begin{aligned}
\left\langle Z, \mathbf{Q} Z^{\prime}\right\rangle & =\left\langle\sum_{p=0}^{n-1} \varepsilon^{-p m} \mathbf{R}^{p} z, \mathbf{Q} \sum_{p^{\prime}=0}^{n-1} \varepsilon^{-p^{\prime} m} \mathbf{R}^{p \prime} z^{\prime}\right\rangle \\
& =\sum_{p=0}^{n-1}\left\langle\varepsilon^{-p m} \mathbf{R}^{p} z, \mathbf{Q} \varepsilon^{-p m} \mathbf{R}^{p} z^{\prime}\right\rangle \\
& =\sum_{p=0}^{n-1}\left\langle z, \mathbf{Q} z^{\prime}\right\rangle \\
& =n\left\langle z, \mathbf{Q} z^{\prime}\right\rangle .
\end{aligned}
$$

We have used the fact that for $p \neq p^{\prime}, \mathbf{R}^{p}$ and $\mathbf{R}^{p^{\prime}}$ send the nonnull parts of $z$ and $z^{\prime}$ on different sectors, so that $\left\langle\mathbf{R}^{p} z, \mathbf{Q R}^{p^{\prime}} z^{\prime}\right\rangle=0$. If $Z=U+i V$ and $Z^{\prime}=U^{\prime}+i V^{\prime}$ (correspondingly, $z=u+i v$ and $z^{\prime}=u^{\prime}+i v^{\prime}$ ), this can be written as

$$
\left\{\begin{array}{l}
\left\langle U, \mathbf{Q} U^{\prime}\right\rangle+\left\langle V, \mathbf{Q} V^{\prime}\right\rangle=n\left(\left\langle u, \mathbf{Q} u^{\prime}\right\rangle+\left\langle v, \mathbf{Q} v^{\prime}\right\rangle\right) \\
\left\langle U, \mathbf{Q} V^{\prime}\right\rangle-\left\langle V, \mathbf{Q} U^{\prime}\right\rangle=n\left(\left\langle u, \mathbf{Q} v^{\prime}\right\rangle-\left\langle v, \mathbf{Q} u^{\prime}\right\rangle\right)
\end{array}\right.
$$

(for any $m$ ) .

Furthermore, as $\left\langle\bar{Z}, \mathbf{Q} Z^{\prime}\right\rangle=\left\langle\mathbf{R} \bar{Z}, \mathbf{Q R} Z^{\prime}\right\rangle=\left\langle\varepsilon^{-m} \bar{Z}\right.$, $\left.\mathbf{Q} \varepsilon^{m} Z^{\prime}\right\rangle=\varepsilon^{2 m}\left\langle\bar{Z}, \mathbf{Q} Z^{\prime}\right\rangle$, we have $\left\langle\bar{Z}, \mathbf{Q} Z^{\prime}\right\rangle=0$ for the coupled problems (i.e., for $m$ such that $\varepsilon^{2 m} \neq 1$ ). So, in this case, we have

$$
\left\{\begin{array}{l}
\left\langle U, \mathbf{Q} U^{\prime}\right\rangle-\left\langle V, \mathbf{Q} V^{\prime}\right\rangle=0 \\
\left\langle U, \mathbf{Q} V^{\prime}\right\rangle+\left\langle V, \mathbf{Q} U^{\prime}\right\rangle=0
\end{array}\left(\text { for } m \text { such as } \varepsilon^{2 m} \neq 1\right) .\right.
$$

Table II summarizes the connection between integral values on the whole domain and on the sector.

If $(U, V)$ is a solution of the coupled problem, then for any $\varepsilon$,

$$
\left\{\begin{array}{l}
U^{\prime}=\mathbf{R}_{\varepsilon} U=U \cos \varepsilon-V \sin \varepsilon \\
V^{\prime}=\mathbf{R}_{\varepsilon} V=U \sin \varepsilon+V \cos \varepsilon
\end{array}\right.
$$

is also a solution, and the functions obtained as a result of the iterative numerical process can drift in the solution plane. Then it is possible that even if the convergence in $k$ (integral convergence) is reached eventually, pointwise functional convergence may never be achieved. To lift indeterminacy in the solution plane, the $U$ and $V$ obtained at the inner iteration stage may be rotated by an angle $\varepsilon$ as above so that $U^{\prime}$ is kept orthogonal to a given function $X$, itself not orthogonal to the solution plane. This condition may be written as $\cot \varepsilon=-\frac{\langle V, X\rangle}{\langle U, X\rangle}$.

\section{IV.C. Filtering Technique for Coupled Problems}

The filtering technique for a higher mode search can be applied straightforwardly to the $\mathbf{R}$-invariant and $\mathbf{R}$-antisymmetric problems. For coupled problems, an extension of this basic technique can be drawn as follows. Assume the fundamental mode and the first $n-1$ forward and adjoint harmonics have been computed for both components of the coupled problem, namely $(i=0, \ldots, n-1)$ :

$$
\begin{gathered}
\left\{\begin{array}{l}
\left(\mathbf{A}-\frac{\mathbf{F}}{k_{i}}\right) U_{i}=0 \\
\left(\mathbf{A}-\frac{\mathbf{F}}{k_{i}}\right) V_{i}=0
\end{array} ;\left\{\begin{array}{l}
\left(\mathbf{A}^{+}-\frac{\mathbf{F}^{+}}{k_{i}}\right) U_{i}^{+}=0 \\
\left(\mathbf{A}^{+}-\frac{\mathbf{F}^{+}}{k_{i}}\right) V_{i}^{+}=0
\end{array} ;\right.\right. \\
\left\{\begin{array} { l } 
{ \mathbf { R } U _ { i } = c _ { m } U _ { i } - s _ { m } V _ { i } } \\
{ \mathbf { R } V _ { i } = s _ { m } U _ { i } + c _ { m } V _ { i } }
\end{array} \text { and } \left\{\begin{array}{l}
\mathbf{R} U_{i}^{+}=c_{m} U_{i}^{+}-s_{m} V_{i}^{+} \\
\mathbf{R} V_{i}^{+}=s_{m} U_{i}^{+}+c_{m} V_{i}^{+}
\end{array}\right.\right.
\end{gathered}
$$

with, for $i \neq j:\left\langle U_{i}^{+}, \mathbf{F} U_{j}\right\rangle=\left\langle U_{i}^{+}, \mathbf{F} V_{j}\right\rangle=\left\langle V_{i}^{+}, \mathbf{F} U_{j}\right\rangle=$ $\left\langle V_{i}^{+}, \mathbf{F} V_{j}\right\rangle=0$. Then the inner iterations yield a calculation result for the $n$ 'th harmonic, this result being possibly contaminated by the previous harmonics:

$$
\left\{\begin{array}{l}
\left(\mathbf{A}-\frac{\mathbf{F}}{k_{n}}\right) U_{n}=0 \\
\left(\mathbf{A}-\frac{\mathbf{F}}{k_{n}}\right) V_{n}=0
\end{array}\right.
$$

and

$$
\left\{\begin{array}{l}
\mathbf{R} U_{n}=c_{m} U_{n}-s_{m} V_{n} \\
\mathbf{R} V_{n}=s_{m} U_{n}+c_{m} V_{n}
\end{array} .\right.
$$

TABLE II

Connection Between Sector and Whole-Domain Integrals

Noncoupled problems $(\mathbf{R} Z= \pm Z)$

Coupled problems $\left(\mathbf{R} Z=\varepsilon^{m} Z\right.$ with $\left.\varepsilon^{2 m} \neq 1\right)$
$V=V^{\prime}=v=v^{\prime}=0$ and $\left\langle U, \mathbf{Q} U^{\prime}\right\rangle=n\left\langle u, \mathbf{Q} u^{\prime}\right\rangle$

$\left\{\left\langle U, \mathbf{Q} U^{\prime}\right\rangle=\left\langle V, \mathbf{Q} V^{\prime}\right\rangle=(n / 2)\left(\left\langle u, \mathbf{Q} u^{\prime}\right\rangle+\left\langle v, \mathbf{Q} v^{\prime}\right\rangle\right)\right.$

$\left\{\left\langle U, \mathbf{Q} V^{\prime}\right\rangle=-\left\langle V, \mathbf{Q} U^{\prime}\right\rangle=(n / 2)\left(\left\langle u, \mathbf{Q} v^{\prime}\right\rangle-\left\langle v, \mathbf{Q} u^{\prime}\right\rangle\right)\right.$ 
A solution orthogonal to all the previous harmonics can then be constructed as

$$
\left\{\begin{array}{l}
\tilde{U}_{n}=U_{n}-\sum_{i=0}^{n-1} \alpha_{i} U_{i}-\sum_{i=0}^{n-1} \beta_{i} V_{i} \\
\tilde{V}_{n}=V_{n}+\sum_{i=0}^{n-1} \beta_{i} U_{i}-\sum_{i=0}^{n-1} \alpha_{i} V_{i}
\end{array}\right.
$$

and

$$
\left\{\begin{array}{l}
\mathbf{R} \tilde{U}_{n}=c_{m} \tilde{U}_{n}-s_{m} \tilde{V}_{n} \\
\mathbf{R} \tilde{\mathbf{V}}_{n}=s_{m} \tilde{U}_{n}+c_{m} \tilde{V}_{n} .
\end{array}\right.
$$

Given the coefficients in the first equation of the left side system of Eq. (11), the coefficients in the second equation have been set so as to make $\tilde{U}_{n}$ and $\tilde{V}_{n}$ obey the coupled problem condition on the right side system; the boundary conditions on a sector are then automatically satisfied.

The values of these coefficients $\alpha_{i}$ and $\beta_{i}$ are given by $(i=0, \ldots, n-1)$ :

$$
\left\{\begin{array}{r}
0=\left\langle U_{i}^{+}, \mathbf{F} \tilde{U}_{n}\right\rangle=\left\langle U_{i}^{+}, \mathbf{F} U_{n}\right\rangle-\left\langle U_{i}^{+}, \mathbf{F} U_{i}\right\rangle \alpha_{i} \\
-\left\langle U_{i}^{+}, \mathbf{F} V_{i}\right\rangle \beta_{i} \\
0=\left\langle V_{i}^{+}, \mathbf{F} \tilde{U}_{n}\right\rangle=\left\langle V_{i}^{+}, \mathbf{F} U_{n}\right\rangle-\left\langle V_{i}^{+}, \mathbf{F} U_{i}\right\rangle \alpha_{i} \\
-\left\langle V_{i}^{+}, \mathbf{F} V_{i}\right\rangle \beta_{i} .
\end{array}\right.
$$

Effectively, the determinant of the system of index $i$ is, using the same derivation as the one that resulted in Eq. $\left(10^{\prime \prime}\right)$ :

$$
\begin{aligned}
\Delta_{i} & =\left\langle U_{i}^{+}, \mathbf{F} U_{i}\right\rangle\left\langle V_{i}^{+}, \mathbf{F} V_{i}\right\rangle-\left\langle U_{i}^{+}, \mathbf{F} V_{i}\right\rangle\left\langle V_{i}^{+}, \mathbf{F} U_{i}\right\rangle \\
& =\left\langle U_{i}^{+}, \mathbf{F} U_{i}\right\rangle^{2}+\left\langle U_{i}^{+}, \mathbf{F} V_{i}\right\rangle^{2},
\end{aligned}
$$

so that $\Delta_{i} \neq 0$ as soon as $\left\langle U_{i}^{+}, \mathbf{F} U_{i}\right\rangle \neq 0$ or $\left\langle U_{i}^{+}, \mathbf{F} V_{i}\right\rangle \neq 0$. A formally similar derivation can be drawn for the adjoint harmonics.

\section{MIRROR SYMMETRY INVARIANCE WITH RESPECT TO THREE ORTHOGONAL PLANES}

We suppose here the system invariant under $p=1,2$, or 3 mirror symmetries with respect to $p$ mutually orthogonal planes. Let $S_{1}, S_{2}$, and $S_{3}$ be the mirror symmetries with respect to the three mutually orthogonal planes $\Pi_{1}$, $\Pi_{2}$, and $\Pi_{3} . S_{1}, S_{2}$, and $S_{3}$ are involutive and commute; their eigenvalues are $\pm 1 . S_{i} S_{j}$ is the order-2 rotation of axis $\Pi_{i} \cap \Pi_{j}$, and $S_{1} S_{2} S_{3}$ the inversion with respect to the point $\Pi_{1} \cap \Pi_{2} \cap \Pi_{3}$.

$S_{1}, S_{2}$, and $S_{3}$ generate $D_{2 h}=\left\{E, S_{1}, S_{2}, S_{3}, S_{1} S_{2}\right.$, $\left.S_{2} S_{3}, S_{3} S_{1}, S_{1} S_{2} S_{3}\right\}$.

$S_{1}$ and $S_{2}$ generate $C_{2 v}=\left\{E, S_{1}, S_{2}, S_{1} S_{2}\right\}$.

$S_{1}$ alone generates $C_{s}=\left\{E, S_{1}\right\}$.

For $p=3$ (this can be easily particularized to $p=2$ or 1), we can define, according to Sec. III, eight projection operators $\mathbf{P}_{\varepsilon_{1} \varepsilon_{2} \varepsilon_{3}}$, which can be cast into the form (each of the $\varepsilon_{j}$ can take the values \pm 1 ):

$$
\mathbf{P}_{\varepsilon_{1} \varepsilon_{2} \varepsilon_{3}}=\frac{1}{8}\left(\mathbf{E}+\varepsilon_{1} \mathbf{S}_{1}\right)\left(\mathbf{E}+\varepsilon_{2} \mathbf{S}_{2}\right)\left(\mathbf{E}+\varepsilon_{3} \mathbf{S}_{3}\right)
$$

The projections of any real function $U$ are eigenfunctions of the mirror symmetries, and $U$ is the sum of its projections:

$$
S_{j}\left(\mathbf{P}_{\varepsilon_{1} \varepsilon_{2} \varepsilon_{3}} U\right)=\varepsilon_{j} \mathbf{P}_{\varepsilon_{1} \varepsilon_{2} \varepsilon_{3}} U \text { and } U=\sum_{\varepsilon_{1}, \varepsilon_{2}, \varepsilon_{3}} \mathbf{P}_{\varepsilon_{1} \varepsilon_{2} \varepsilon_{3}} U
$$

Assume $U$ to be simultaneously an eigenfunction of the symmetry operators $\mathbf{S}_{1}, \mathbf{S}_{2}$, and $\mathbf{S}_{3}$, and a solution of the balance equation over the whole spatial domain:

$$
\mathbf{S}_{j} U=\varepsilon_{j} U \text { and }\left(\mathbf{A}-\frac{\mathbf{F}}{k}\right) U=0,
$$

with a given boundary condition for the whole system. The restriction $u$ of $U$ to the octant $O$ limited by the planes $\Pi_{1}, \Pi_{2}$, and $\Pi_{3}$ is such that

$$
\begin{array}{ll}
\left(\mathbf{A}-\frac{\mathbf{F}}{k}\right) u=0, & \text { on octant } O ; \\
u=0, & \text { outside octant } O ; \\
\begin{array}{l}
\text { Boundary condition of the global problem, } \\
u\left(S_{j} \omega\right)=\varepsilon_{j} u(\omega),
\end{array} & \begin{array}{l}
\text { boundaries other than the plan } \\
\text { on plane } \Pi_{j}(j=1,2,3) .
\end{array}
\end{array}
$$

The boundary condition for the $\mathbf{S}_{j}$-invariant problems $\left(\varepsilon_{j}=+1\right)$ is the usual mirror symmetry condition. To our knowledge, the condition for the $\mathbf{S}_{j}$-antisymmetric problems $\left(\varepsilon_{j}=-1\right)$ has been used only occasionally, e.g., it is the antireflective boundary condition in Ref. 30.

$U$ (on the whole spatial domain) can be reconstructed from $u$ (on octant $O$ ) as

$$
U=\left(\mathbf{E}+\varepsilon_{1} \mathbf{S}_{1}\right)\left(\mathbf{E}+\varepsilon_{2} \mathbf{S}_{2}\right)\left(\mathbf{E}+\varepsilon_{3} \mathbf{S}_{3}\right) u .
$$


It can be checked that $\mathbf{S}_{j} U=\varepsilon_{j} U$ and that the boundary conditions (13) ensure continuity of $Z$ at octant faces. Integrals such as $\left\langle U, \mathbf{Q} U^{\prime}\right\rangle$, where $U$ and $U^{\prime}$ are solutions of the balance equation that are eigenfunctions of the $\mathbf{S}$ for the same eigenvalues $\varepsilon_{j}$ and $\mathbf{Q}$ is any operator commuting with the $\mathbf{S}_{j}$, can be expressed using integrals only involving the solutions $u$ and $u^{\prime}$ on octant $O$ [we use that $U$ and $U^{\prime}$ are expressed by Eq. (14) as linear combinations of eight operators sending each nonnull part of $u$ and $u^{\prime}$ on a different octant]:

$$
\left\langle U, \mathbf{Q} U^{\prime}\right\rangle=8\left\langle u, \mathbf{Q} u^{\prime}\right\rangle .
$$

\section{SYSTEMS INVARIANT UNDER MIRROR SYMMETRIES AND DISCRETE ROTATIONS}

We restrict again to prismatic systems of vertical axis $\Delta$ but assume there are vertical mirror symmetry planes containing $\Delta$. If there are two such vertical mirror symmetry planes $\Pi_{0}$ and $\Pi_{1}$ separated by an angle $\pi / n(n>1)$ and corresponding to mirror symmetries $S_{0}$ and $S_{1}, R=$ $S_{0} S_{1}$ is the rotation of axis $\Delta$ and angle $2 \pi / n$ (as an illustration, see Fig. 2, established for hexagonal geometry) and the group generated by $S_{0}$ and $S_{1}$ is the dihedral group $C_{n v}=\left\{E, R, R^{2}, \ldots, R^{n-1}, S_{0}, S_{1}, S_{2}, \ldots, S_{n-1}\right\}$ with $S_{p}=S_{0} R^{p}$ and the following composition rules (index sum or difference taken modulo $n$ ):

$$
\left\{\begin{array}{rlrl}
R^{p} R^{q} & =R^{p+q} & & S_{p} R^{q}=S_{p+q} \\
S_{p} S_{q} & =R^{q-p} & & R^{p} S_{q}=S_{q-p}
\end{array} .\right.
$$

Note that as a particular case, $R S_{p}=S_{p} R^{-1}=S_{p-1}$.

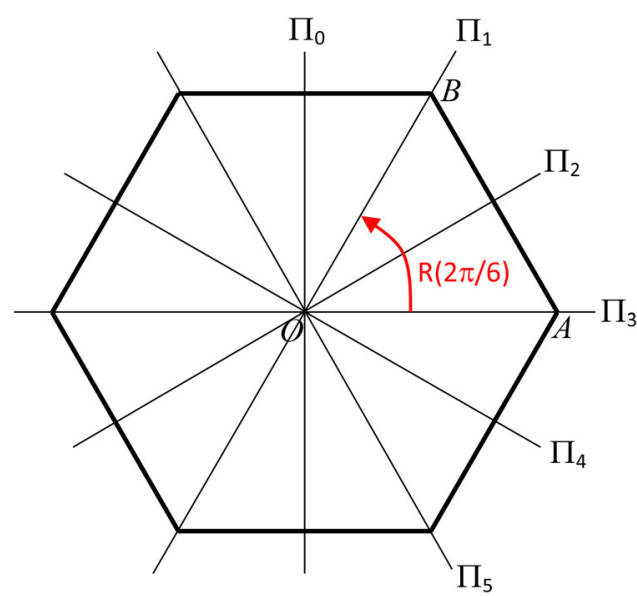

Fig. 2. Cross section of a prismatic hexagonal homogeneous system with a trace of the mirror symmetry planes $\left(\Pi_{0}\right.$ to $\Pi_{5}$ ), with $\pi / 6$ clockwise angle between one and the next. $R=S_{0} S_{1}$ is the counterclockwise rotation of angle $2 \pi / 6$.
We cannot solve on a sector of angle $\pi / n(n>2)$ using only reflective boundary conditions at left and right edges, as the functions so obtained would be invariant or antisymmetric for mirror symmetries with respect to the edges of the sector and for their product, the rotation of angle $2 \pi / n$ : all solutions related to the complex eigenvalues of this rotation (the coupled problems) would then be skipped. When the system is $C_{n v}$-invariant ( $n>2$ ), we can restrict resolution of the equations over a $2 \pi / n$ sector only when using rotation-related boundary conditions. Anyway, once having found a $k$-eigenfunction using one classification type (related either to rotations on a $2 \pi / n$ sector or to mirror symmetries with respect to 1,2 , or 3 orthogonal planes), then applying projections of the other type to it will possibly (not always) yield directly additional linearly independent $k$-eigenfunctions.

\section{VI.A. Solving First for Rotation Eigenfunctions}

If $\alpha$ is an $\mathbf{R}$-invariant $k$-eigenfunction not belonging to a $\mathbf{S}_{0}$-eigenspace, it can be projected onto the two orthogonal $\mathbf{S}_{0}$-eigenspaces, yielding the $\mathbf{R}$-invariant independent functions $a=\frac{1}{2}\left(\alpha+\mathbf{S}_{0} \alpha\right)$ and $\tilde{a}=\frac{1}{2}\left(\alpha-\mathbf{S}_{0} \alpha\right)$, such as $\mathbf{S}_{i} a=a, \mathbf{S}_{i} \tilde{a}=-\tilde{a}$, and $\langle a, \mathbf{Q} \tilde{a}\rangle=0$ for any $\mathrm{C}_{\mathrm{nv}^{-}}$ invariant operator $\mathbf{Q}$.

If $\beta$ is an $\mathbf{R}$-antisymmetric $k$-eigenfunction not belonging to a $\mathbf{S}_{0}$-eigenspace, it can be projected onto the two orthogonal $\mathbf{S}_{0}$-eigenspaces, yielding the $\mathbf{R}$-antisymmetric independent functions $b=\frac{1}{2}\left(\beta+\mathbf{S}_{0} \beta\right)$ and $\tilde{b}=\frac{1}{2}(\beta-$ $\left.\mathbf{S}_{0} \beta\right)$ such as $\mathbf{S}_{i} b=b, \mathbf{S}_{i} \tilde{b}=-\tilde{b}$, and $\langle b, \mathbf{Q} \tilde{b}\rangle=0$ for any $\mathrm{C}_{\mathrm{nv}}$-invariant operator $\mathbf{Q}$.

For the coupled problems, assume a function couple $\left(\varphi_{m}, \psi_{m}\right)$ such as $\mathbf{R}\left(\varphi_{m}+i \psi_{m}\right)=\varepsilon^{m}\left(\varphi_{m}+i \psi_{m}\right)$ has been found. Then, multiplying by $-i, \mathbf{R}\left(\psi_{m}-i \varphi_{m}\right)=$ $\varepsilon^{m}\left(\psi_{m}-i \varphi_{m}\right)$, and so, $\left(\psi_{m},-\varphi_{m}\right)$ is also a solution of the coupled problem. Using the composition rules,

$$
\begin{gathered}
\mathbf{R S}_{p}\left(\varphi_{m}-i \psi_{m}\right)=\mathbf{S}_{p} \mathbf{R}^{-1}\left(\varphi_{m}-i \psi_{m}\right) \\
=\mathbf{S}_{p} \overline{\mathbf{R}^{-1}\left(\varphi_{m}+i \psi_{m}\right)}=\mathbf{S}_{p} \overline{\varepsilon^{-m}\left(\varphi_{m}+i \psi_{m}\right)} \\
=\varepsilon^{m} \mathbf{S}_{p}\left(\varphi_{m}-i \psi_{m}\right) .
\end{gathered}
$$

This means that $\left(\mathbf{S}_{p} \varphi_{m},-\mathbf{S}_{p} \psi_{m}\right)$ is also a solution of the coupled problem. Finally, combining these two results, $\left(\mathbf{S}_{p} \psi_{m}, \mathbf{S}_{p} \varphi_{m}\right)$ is another solution of the coupled problem. These relations can be used to construct formally two solution couples of the coupled problems with invariance properties with respect to $\mathbf{S}_{p}$, e.g., with the first component of each couple being $\mathbf{S}_{p}$-invariant and the second $\mathbf{S}_{p}$-antisymmetric: 


$$
\left\{\begin{array}{l}
u_{m}=\boldsymbol{\sigma}_{p}^{+} \varphi_{m}=\frac{1}{2}\left(\varphi_{m}+\mathbf{S}_{p} \varphi_{m}\right) \\
v_{m}=\boldsymbol{\sigma}_{p}^{-} \psi_{m}=\frac{1}{2}\left(\psi_{m}-\mathbf{S}_{p} \psi_{m}\right)
\end{array}\right.
$$

and

$$
\left\{\begin{array}{l}
\tilde{u}_{m}=\boldsymbol{\sigma}_{p}^{+} \psi_{m}=\frac{1}{2}\left(\psi_{m}+\mathbf{S}_{p} \psi_{m}\right) \\
\tilde{v}_{m}=-\boldsymbol{\sigma}_{p}^{-} \varphi_{m}=\frac{1}{2}\left(-\varphi_{m}+\mathbf{S}_{p} \varphi_{m}\right) .
\end{array}\right.
$$

It is easily checked that if $\left(\varphi_{m}, \psi_{m}, \mathbf{S}_{p} \varphi_{m}, \mathbf{S}_{p} \psi_{m}\right)$ are linearly independent, so are $\left(u_{m}, v_{m}, \tilde{u}_{m}, \tilde{v}_{m}\right)$; in this case, the two solutions initially found yield, in fact, four independent solutions, thus sparing calculation time. In contrast, if $\left(\varphi_{m}, \psi_{m}, \mathbf{S}_{p} \varphi_{m}, \mathbf{S}_{p} \psi_{m}\right)$ are linearly dependent, they span a plane only and no additional independent solution can be found this way. A practical check of the coplanarity of $u, v$, $\mathbf{S}_{p} u$, and $\mathbf{S}_{p} v$ may be implemented as follows: if $u, v$, and $\mathbf{S}_{p} u$ are coplanar, then $\langle u, u\rangle \mathbf{S}_{p} u=\left\langle u, \mathbf{S}_{p} u\right\rangle u+\left\langle v, \mathbf{S}_{p} u\right\rangle v$, as $u$ and $v$ are orthogonal and of same norm, and by squaring: $\langle u, u\rangle^{2}=\left\langle u, \mathbf{S}_{p} u\right\rangle^{2}+\left\langle v, \mathbf{S}_{p} u\right\rangle^{2}$.

\section{VI.B. Solving First for Mirror Symmetry Eigenfunctions}

Assume we have found a real solution $f$ of the problem within a class of reflective boundary conditions; we can apply to it the projectors on $\mathbf{R}$-invariant subspaces: $\mathbf{u}_{m}$ and $\mathbf{v}_{m}(0 \leq m \leq n / 2)$. If $f$ already belongs to one of these R-invariant subspaces, all projections on any other R-invariant subspace will yield null functions (if $f$ belongs to the $m$ 'th coupled problem $\mathbf{R}$-invariant subspace, application of $\mathbf{u}_{m}$ and $\mathbf{v}_{m}$ will produce an additional linearly independent $k$-eigenfunction). If not, and if degeneracy extends over more than one $\mathbf{R}$-invariant subspace, several new linearly independent $k$-eigenfunctions may be produced directly this way from $f$.

\section{TWO SIMPLE ILLUSTRATIVE EXAMPLES}

These examples are given to illustrate on simple configurations the validity of the previous theoretical derivations, and how the possibly degenerated $k$-eigenfunctions may be sorted into the symmetry classes belonging to commutative subgroups of the symmetry group. They involve monoenergetic diffusion over simple geometric objects made of a homogeneous material. Looking for $k$-eigenfunctions of the diffusion equation is then equivalent to searching for eigenfunctions of the Laplacian operator. The boundary condition used will be a null flux on the outer boundary (Dirichlet condition). Two examples are given, involving the symmetry groups $C_{3 v}$ (equilateral triangle) and $C_{6 v}$ (regular hexagon), respectively. The homogeneous square geometry (symmetry group $C_{4 v}$ ), also with an analytic solution, will be used in the companion paper $^{32}$ as a simple validation tool to check the implementation, in a diffusion solver and rectangular geometry, of innovative boundary conditions with a solution over a sector.

\section{VII.A. Equilateral Triangle, Symmetry Group $C_{3 v}$}

This problem has an analytical solution. ${ }^{33}$ Most of the result is in the Appendix for the interested reader. A complete orthogonal basis of analytic solutions of equation $\Delta T+\chi^{2} T=0$ on the homogeneous equilateral triangle vanishing on the sides is

$$
\left\{T_{s}^{m n}\right\}_{0<m \leq n} \cup\left\{T_{a}^{m n}\right\}_{0<m<n} .
$$

$T_{s}^{m n}$ and $T_{a}^{m n}$ (see formulation and properties in the Appendix) are, respectively, symmetric and antisymmetric with respect to a given median of the triangle (the vertical median in Fig. 3). Eigenvalues are given by $\chi^{2}=\frac{4 \pi^{2}}{27 r^{2}}\left(m^{2}+m n+n^{2}\right) \cdot R$ being defined as a rotation of angle $2 \pi / 3$, the R-invariant basis functions are those with indices such as $m \equiv n$ (modulo 3 ), while the basis functions with indices not congruent modulo 3 are solutions of the coupled problem for $\mathbf{R}$ :

$$
\left\{\begin{array}{l}
\mathbf{R} T_{s}^{m n}=-\frac{1}{2} T_{s}^{m n}-\frac{\sqrt{3}}{2} T_{a}^{m n} \\
\mathbf{R} T_{a}^{m n}=\frac{\sqrt{3}}{2} T_{s}^{m n}-\frac{1}{2} T_{a}^{m n}
\end{array}\right.
$$

( $m$ and $n$ not congruent modulo 3 ) .

The multiplicity of an eigenvalue $\chi^{2}=\frac{4 \pi^{2}}{27 r^{2}} q$ depends on the number of possible representations of $q$ by the quadratic form $m^{2}+m n+n^{2}$ with $m \leq n$. If only one such representation is possible, the behavior is given in Table III (the presence of two conjugate complex $\mathbf{R}$-eigenvalues means a real coupled problem with an R-invariant plane).

The mirror symmetries double the degeneracy with respect to the pure rotation case for $m \neq n$ with $m$ congruent to $n$ modulo 3 , as we have then only one rotation eigenvalue $(+1)$ for a two-dimensional invariant subspace, and so two linearly independent $\mathbf{R}$-invariant basis eigenfunctions, one symmetric and the other antisymmetric with respect to the chosen median. In that case, one calculation may provide two eigenfunctions thanks to the projection operators $\mathbf{P}_{+1}$ and $\mathbf{P}_{-1}$ (see Sec. V).

Figure 3 illustrates some examples. The fundamental mode corresponds to $(m, n)=(1,1)$ and is an $\mathbf{R}$-invariant 


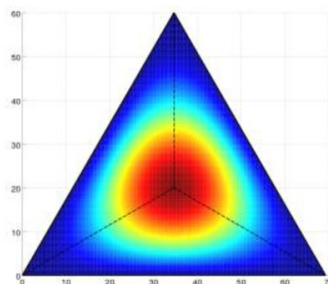

$$
\begin{aligned}
& (\mathrm{m}, \mathrm{n})=(1,1) \\
& \varepsilon_{\text {Rot }}=+1 \\
& \varepsilon_{\text {Sym }}=+1
\end{aligned}
$$

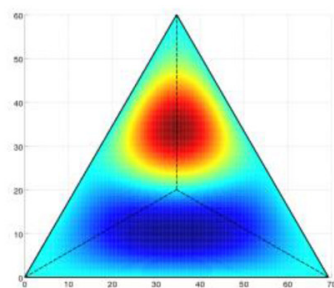

$(m, n)=(1,2)$

$\varepsilon_{\text {Rot }}=e^{ \pm \frac{2 i \pi}{3}}$

$\varepsilon_{\text {Sym }}=+1$

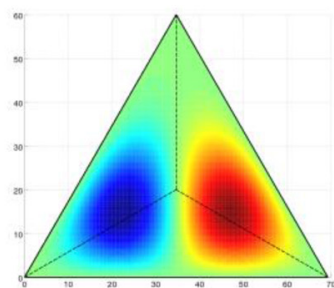

$(m, n)=(1,2)$

$\varepsilon_{\text {Rot }}=e^{ \pm \frac{2 i \pi}{3}}$

$\varepsilon_{\text {Sym }}=-1$

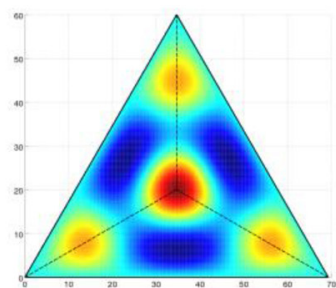

$(m, n)=(1,4)$

$\varepsilon_{\text {Rot }}=+1$

$\varepsilon_{\text {Sym }}=+1$

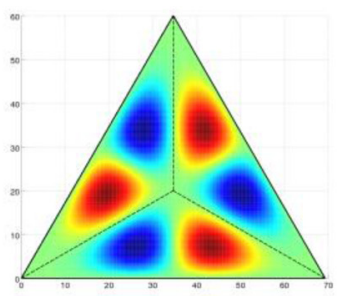

$(m, n)=(1,4)$

$\varepsilon_{\text {Rot }}=+1$

$\varepsilon_{\text {Sym }}=-1$

Fig. 3. Some eigenfunctions of the Laplacian operator in the equilateral triangle.

TABLE III

Degeneracy of Laplacian Eigenvalues in the Equilateral Triangle and Projection of Eigenfunctions on $C_{3 v}$-Invariant Subspaces

\begin{tabular}{|c|c|c|c|c|}
\hline \multirow{2}{*}{} & \multirow{2}{*}{$\begin{array}{c}\text { Dimension of Invariant } \\
\chi^{2} \text {-Eigenspace }\end{array}$} & \multicolumn{2}{|c|}{$\begin{array}{c}\text { Eigenvalues in Invariant } \\
\chi^{2} \text {-Eigenspace for } \ldots\end{array}$} \\
\cline { 3 - 5 } & & 1 & +1 & +1 \\
\hline$m=n$ & Rotation & Mirror Symmetries \\
\hline \multirow{2}{*}{$m \neq n$} & Congruent modulo 3 & 2 & +1 \\
$e^{ \pm \frac{2 i \pi}{3}}$ & \pm 1 \\
& Not congruent modulo 3 & 2 & \pm 1 \\
\hline
\end{tabular}

function. For higher-order eigenfunctions, when $m$ is not congruent to $n$ modulo 3, the twofold degeneracy is already present in the pure rotation case (coupled problem), and the mirror symmetries can provide no additional linearly independent eigenfunction; they only allow us to shape the solutions of the coupled problem into a symmetric and an antisymmetric solution in the same invariant plane. This is the case for $(m, n)=(1,2)$. The degeneracy is doubled only for $\mathbf{R}$-invariant solutions with $m \neq n$ while $m$ is congruent to $n$ modulo 3, for instance with $(m, n)=(1,4)$.

\section{VII.B. Hexagon: Classifications According to Rotations or Mirror Symmetries}

We take here the example of the homogeneous regular hexagonal prism of vertical axis (see Fig. 2) with six mirror symmetry planes ( $\Pi_{0}$ to $\Pi_{5}$, associated with mirror symmetries $S_{0}$ to $S_{5}$ ) and, consequently, invariance under the order- 6 rotation $R$. The system is invariant under the action of the noncommutative symmetry group $C_{6 v}=\left\{I, R, R^{2}, R^{3}, R^{4}, R^{5}, S_{0}, S_{1}, S_{2}, S_{3}, S_{4}, S_{5}\right\}$.

Using only the material rotation invariance under $R$, i.e., under the action of the cyclic (commutative) subgroup $C_{6}=\left\{I, R, R^{2}, R^{3}, R^{4}, R^{5}\right\}$, we search for $k$-eigenfunctions in $\mathbf{R}$-invariant subspaces corresponding to eigenvalues $e^{m \frac{2 i \pi}{6}}$ for $0 \leq m \leq 3$, resulting in the four solutions classes below:

$$
\begin{aligned}
& \mathbf{R} a=a, \\
& \left\{\begin{array}{l}
\mathbf{R} u=\frac{1}{2} u-\frac{\sqrt{3}}{2} v \\
\mathbf{R} v=\frac{\sqrt{3}}{2} u+\frac{1}{2} v, \\
\mathbf{R} u^{\prime}=-\frac{1}{2} u^{\prime}-\frac{\sqrt{3}}{2} v^{\prime} \\
\mathbf{R} v^{\prime}=\frac{\sqrt{3}}{2} u^{\prime}-\frac{1}{2} v^{\prime},
\end{array}\right.
\end{aligned}
$$

and

$$
\mathbf{R} b=-b .
$$

It is then easy to check that

$$
\left\{\begin{array}{l}
b+\mathbf{R}^{3} b=u+\mathbf{R}^{3} u=v+\mathbf{R}^{3} v=0 \\
a-\mathbf{R}^{3} a=u^{\prime}-\mathbf{R}^{3} u^{\prime}=v^{\prime}-\mathbf{R}^{3} v^{\prime}=0 .
\end{array}\right.
$$

If, in addition to $R$, we use any given mirror symmetry (e.g., $S_{0}$ ), shifting to the full symmetry group $C_{6 v}$, degeneracy inside a given class may be doubled if the 
$\mathbf{R}$-eigenvector found (real or complex) and its image by $\mathbf{S}_{0}$ are linearly independent. In any case, we assume we have obtained for the coupled problems solutions such as $\left\{\begin{array}{l}\mathbf{S}_{0} u=u \\ \mathbf{S}_{0} v=-v\end{array}\right.$ and $\left\{\begin{array}{l}\mathbf{S}_{0} u^{\prime}=u^{\prime} \\ \mathbf{S}_{0} v^{\prime}=-v^{\prime}\end{array}\right.$ (see end of Sec. VI.A). The resulting partition of solutions into four rotation classes is given in Table IV. For the $m=0$ and $m=3$ classes, functions with either symmetry or antisymmetry with respect to $\mathbf{S}_{0}$ may exist. If, instead, we start by taking into account two mirror symmetries with respect to perpendicular planes (e.g., $S_{0}$ and $S_{3}$ ), thus using effectively the subgroup $C_{2 v}=\left\{I, R^{3}, S_{0}, S_{3}\right\}$ to partition the problem into four classes, then these classes contain functions with $\mathbf{R}$-invariance properties as given in Table $\mathrm{V}$. Using the relation $\mathbf{R}^{3} \mathbf{S}_{0}=\mathbf{S}_{0} \mathbf{R}^{3}=\mathbf{S}_{3}$, it follows that $\left\{\begin{array}{l}\mathbf{S}_{3} u=-u \\ \mathbf{S}_{3} v=v\end{array}\right.$ and $\left\{\mathbf{S}_{3} u^{\prime}=u^{\prime}\right.$ $\left\{\mathbf{S}_{3} v^{\prime}=-v^{\prime}\right.$

Here the degeneracy of the coupled problems is lifted, in the sense that the solutions of the functions of the coupled problems are split into different classes.

\section{CONCLUSIONS}

In neutron chain systems with material symmetries, various $k$-eigenvalues of the neutron balance equation beyond the dominant one are degenerate, i.e., with several

\section{TABLE IV}

Partition of the Regular Hexagon Eigenfunctions into Symmetry Classes According to Order-6 Rotation $R$ and Mirror Symmetry $S_{0}$

\begin{tabular}{|l|c|c|}
\hline & $\mathbf{S}_{0}$-symmetric & $\mathbf{S}_{0}$-antisymmetric \\
\hline R-invariant, $m=0$ & \multicolumn{2}{|c|}{$\mathbf{R} a=a$} \\
\hline Coupled problem, $m=1$ & $u \in(u, v)$ & $v \in(u, v)$ \\
\hline Coupled problem, $m=2$ & $u^{\prime} \in\left(u^{\prime}, v^{\prime}\right)$ & $v^{\prime} \in\left(u^{\prime}, v^{\prime}\right)$ \\
\hline R-antisymmetric, $m=3$ & \multicolumn{2}{|c|}{$\mathbf{R} b=-b$} \\
\hline
\end{tabular}

TABLE V

Partition of the Regular Hexagon Eigenfunctions into Symmetry Classes According to Mirror Symmetries $S_{0}$ and $S_{3}$ with Respect to Perpendicular Planes

\begin{tabular}{|l|c|c|}
\hline & $\mathbf{S}_{3}$-symmetric & $\mathbf{S}_{3}$-antisymmetric \\
\hline $\mathbf{S}_{0}$-symmetric & $\begin{array}{c}\mathbf{R} a=a \\
u^{\prime} \in\left(u^{\prime}, v^{\prime}\right)\end{array}$ & $\begin{array}{c}\mathbf{R} b=-b \\
u \in(u, v)\end{array}$ \\
\hline $\mathbf{S}_{0}$-antisymmetric & $\begin{array}{c}\mathbf{R} b=-b \\
v \in(u, v)\end{array}$ & $\begin{array}{c}\mathbf{R} a=a \\
v^{\prime} \in\left(u^{\prime}, v^{\prime}\right)\end{array}$ \\
\hline
\end{tabular}

linearly independent eigenfunctions associated with the same eigenvalue $k$.

It has been shown here that in such cases, the various eigenfunctions of the neutron balance equation associated with the same eigenvalue can be partitioned into several symmetry classes according to their invariance properties with respect to the symmetry operations, leaving the material distribution unchanged in the system. Furthermore, the calculation of these eigenfunctions can be limited to a fraction of the system (sector) provided that boundary conditions matching the symmetry operations are used. The wholesystem eigenfunctions can then be deduced (unfolded) from the solutions obtained over the sector.

If there is material invariance under $p=1,2$, or 3 mirror symmetries with respect to mutually orthogonal planes, the balance equation can be solved on a sector representing the fraction $1 / 2^{p}$ of the system; the boundary conditions are of the reflective type, either symmetric (the most usual) or antisymmetric (seldom considered in the past), effectively partitioning the solutions into $2^{p}$ classes with respect to the boundary condition type for each symmetry plane.

If there is material invariance under a rotation $R$ of order $n$, the balance equation can be solved on a sector representing the fraction $1 / n$ of the system; the boundary conditions link matching phase space elements of the left and right boundaries of the sector while the whole-system boundary conditions are still applied to the outer boundary of the sector. The calculation is partitioned into several class types:

\section{R-invariant functions}

2. R-antisymmetric functions (only for even $n$ )

3. as many classes of coupled functions (real and imaginary parts of complex R-eigenfunctions) as there are integers $m$ satisfying $1<m<n / 2$.

To our knowledge, the boundary conditions involved here (except for the basic R-invariance) do not seem to have been addressed yet.

If there is material invariance under rotations and mirror symmetries, either approach may be used, resulting in different classifications of the eigenfunctions.

When using the power iteration method to search $k$-eigenvalues, the use of material symmetries to split the global problem into a variety of smaller-sized problems has several computational advantages:

1. Each class of solutions is worked out separately on only a fraction of the global system, allowing a parallel treatment of the various classes.

2 . The whole $k$-spectrum is partitioned into the various classes, increasing the dominance ratios in each class with respect to the whole-core problem. 
3. The degeneracy of any given $k$-eigenvalue may also be partitioned into the symmetry classes.

\section{APPENDIX}

\section{ANALYTICAL SOLUTION FOR MONOENERGETIC DIFFUSION IN THE EQUILATERAL TRIANGLE}

We consider an equilateral triangle with inradius $r$, and Lamé's triangular coordinates $u, v$, and $w$ (see Fig. A.1), such that $u+2 r, v+2 r$, and $w+2 r$ are the distances of a generic point of the triangle to the parallels of the sides drawn by each vertex. The center of the triangle has coordinates $(0,0,0)$, and $u, v$, and $w$ are linked by the condition $u+v+w=0$.

Lamé obtained analytical solutions, symmetric or antisymmetric with respect to the $u$-axis of equation $v=w$ and obeying Dirichlet conditions on the sides. They are given by the following formulas, the three integer indices being linked by $l+m+n=0$ :

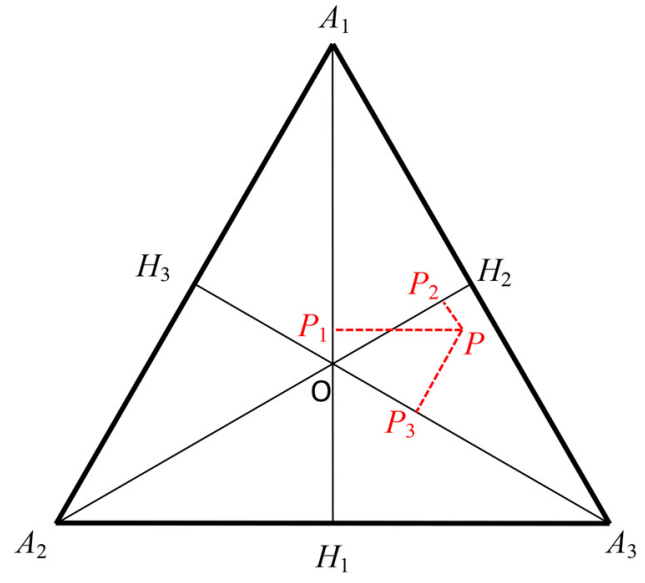

Fig. A.1. Lamé's coordinates of point $P$ in the equilateral triangle are such that $u=A_{1} P_{1}-2 r, v=A_{2} P_{2}-2 r$, and $w=A_{3} P_{3}-2 r\left(r\right.$ being the inradius of the triangle and $P_{1}$, $P_{2}$, and $P_{3}$ being, respectively, the orthogonal projections of $P$ onto $A_{1} H_{1}, A_{2} H_{2}$, and $\left.A_{3} H_{3}\right)$. Examples are $O(0,0,0)$, $A_{1}(-2 r, 0,0)$, and $H_{1}(r,-r / 2,-r / 2)$.

and

$$
\begin{gathered}
T_{s}^{m n}=\sin \left[\frac{\pi l}{3 r}(u+2 r)\right] \cos \left[\frac{\pi(m-n)}{9 r}(v-w)\right]+\sin \left[\frac{\pi m}{3 r}(u+2 r)\right] \cos \left[\frac{\pi(n-l)}{9 r}(v-w)\right] \\
+\sin \left[\frac{\pi n}{3 r}(u+2 r)\right] \cos \left[\frac{\pi(l-m)}{9 r}(v-w)\right]
\end{gathered}
$$

$$
\begin{aligned}
T_{a}^{m n}=\sin \left[\frac{\pi l}{3 r}(u+2 r)\right] & \sin \left[\frac{\pi(m-n)}{9 r}(v-w)\right]+\sin \left[\frac{\pi m}{3 r}(u+2 r)\right] \sin \left[\frac{\pi(n-l)}{9 r}(v-w)\right] \\
& +\sin \left[\frac{\pi n}{3 r}(u+2 r)\right] \sin \left[\frac{\pi(l-m)}{9 r}(v-w)\right] .
\end{aligned}
$$

These functions vanish on the sides of the triangle and are such that $\Delta T_{s / a}^{m n}+k^{2} T_{s / a}^{m n}=0$ with $k^{2}=\frac{2 \pi^{2}}{27 r^{2}}\left(l^{2}+\right.$ $\left.m^{2}+n^{2}\right)=\frac{4 \pi^{2}}{27 r^{2}}\left(m^{2}+m n+n^{2}\right)$. They also have the following properties, with the inner product defined as $\langle\varphi, \psi\rangle=\int \varphi \psi d A, d A$ being the area element in the triangle:

1. $T_{s}^{m n}$ vanishes when one of the integers $l, m$, or $n$ is null; $T_{s}^{m n}=T_{s}^{n m}$.

2. $T_{a}^{m n}$ vanishes when one of the integers $l, m$, or $n$ is null or if two of them are equal; $T_{a}^{m n}=-T_{a}^{n m}$.

3. $\left\{T_{s}^{m n}\right\}_{n \geq m>0} \cup\left\{T_{a}^{m n}\right\}_{n>m>0}$ is a complete orthogonal basis of functions over the triangle.

4. $\left\langle T_{s}^{m m}, T_{s}^{m m}\right\rangle=\frac{9 \sqrt{3}}{2} r^{2}$ and $\left\langle T_{s}^{m n}, T_{s}^{m n}\right\rangle=\left\langle T_{a}^{m n}, T_{a}^{m n}\right\rangle=\frac{9 \sqrt{3}}{4} r^{2}(m \neq n)$.

5. $\left\langle 1, T_{s}^{m n}\right\rangle=\frac{9 \sqrt{3}}{\pi m} r^{2} \delta_{m n}$.

6. Condition for invariance under rotation $R$ of angle $2 \pi / 3$ :

a. $\mathbf{R} T_{s}^{m n}=T_{s}^{m n} \Leftrightarrow m \equiv n \quad$ (modulo 3)

b. $\mathbf{R} T_{a}^{m n}=T_{a}^{m n} \Leftrightarrow m \equiv n \quad$ (modulo 3).

An extensive review of the results above, with a historical background dating back to Lamé's pioneering work, can be found in Ref. 33, together with fully worked or sketched proofs. 
The symmetry group of the equilateral triangle is $C_{3 v}=\left\{E, R, R^{2}, S_{0}, S_{1}, S_{2}\right\}$, the symmetry operations being defined by their action on the coordinates as

$$
\begin{array}{ll}
E:(u, v, w) \rightarrow(u, v, w) & S_{0}=S_{u}:(u, v, w) \rightarrow(u, w, v) \\
R:(u, v, w) \rightarrow(v, w, u) & S_{1}=S_{w}:(u, v, w) \rightarrow(v, u, w) \\
R^{2}:(u, v, w) \rightarrow(w, u, v) & S_{2}=S_{v}:(u, v, w) \rightarrow(w, v, u),
\end{array}
$$

in such a way that their composition rules are (sums of indices to be taken modulo 3)

$$
\left\{\begin{array}{l}
R^{p} R^{q}=R^{p+q} \quad S_{p} R^{q}=S_{p+q} \\
S_{p} S_{q}=R^{q-p} \quad R^{p} S_{q}=S_{q-p}
\end{array} .\right.
$$

The following results are easily checked:

$$
\begin{array}{ll}
\mathbf{S}_{0} T_{s}^{m n}=T_{s}^{m n}, & \mathbf{S}_{0} T_{a}^{m n}=-T_{a}^{m n}, \\
\mathbf{S}_{1} T_{s}^{m n}=\mathbf{R}^{2} T_{s}^{m n}, & \mathbf{S}_{1} T_{a}^{m n}=-\mathbf{R}^{2} T_{a}^{m n}, \\
\mathbf{S}_{2} T_{s}^{m n}=\mathbf{R} T_{s}^{m n}, & \mathbf{S}_{2} T_{a}^{m n}=-\mathbf{R} T_{a}^{m n}, \\
\left\langle T_{s}^{m n}, \mathbf{R} T_{s}^{m n}\right\rangle=\left\langle T_{s}^{m n}, \mathbf{R}^{2} T_{s}^{m n}\right\rangle, \\
\left\langle T_{a}^{m n}, \mathbf{R} T_{a}^{m n}\right\rangle=\left\langle T_{a}^{m n}, \mathbf{R}^{2} T_{a}^{m n},,\right.
\end{array}
$$

and

$$
\begin{gathered}
\left\langle T_{s}^{m n}, \mathbf{R} T_{a}^{m n}\right\rangle=\left\langle\mathbf{S}_{0} T_{s}^{m n}, \mathbf{S}_{0} \mathbf{R} T_{a}^{m n}\right\rangle \\
=\left\langle\mathbf{S}_{0} T_{s}^{m n}, \mathbf{R}^{-1} \mathbf{S}_{0} T_{a}^{m n}\right\rangle=-\left\langle\mathbf{R} T_{s}^{m n}, T_{a}^{m n}\right\rangle .
\end{gathered}
$$

Additionally, accurate numerical calculations strongly suggest (and we will assume) that for $m$ and $n$ not congruent modulo 3:

1. $\left\langle\mathbf{R} T_{s}^{m n}, T_{s}^{m n}\right\rangle=\left\langle\mathbf{R} T_{a}^{m n}, T_{a}^{m n}\right\rangle=-\frac{9 \sqrt{3}}{8} r^{2}$.

2. $\left\langle\mathbf{R} T_{s}^{m n}, T_{a}^{m n}\right\rangle=-\left\langle T_{s}^{m n}, \mathbf{R} T_{a}^{m n}\right\rangle=\frac{27}{8} r^{2} \operatorname{sgn}(m-n)$.

These numerical values can be used to show that when $m$ and $n$ are not congruent modulo 3: $\| \mathbf{R} T_{s}^{m n}+\frac{1}{2} T_{s}^{m n}+$ $\frac{\sqrt{3}}{2} T_{a}^{m n} \|=0$ and $\left\|\mathbf{R} T_{a}^{m n}-\frac{\sqrt{3}}{2} T_{s}^{m n}+\frac{1}{2} T_{a}^{m n}\right\|=0$.

In other words, if $m$ and $n$ not congruent modulo 3, then $\left(T_{s}^{m n}, T_{a}^{m n}\right)$ is a solution of the coupled problem

$$
\left\{\begin{array}{l}
\mathbf{R} T_{s}^{m n}=-\frac{1}{2} T_{s}^{m n}-\frac{\sqrt{3}}{2} T_{a}^{m n} \\
\mathbf{R} T_{a}^{m n}=\frac{\sqrt{3}}{2} T_{s}^{m n}-\frac{1}{2} T_{a}^{m n} .
\end{array}\right.
$$

\section{References}

1. S. CARNEY et al., "Theory and Application of the Fission Matrix Method for Continuous-Energy Monte-Carlo," Ann. Nucl. Energy, 73, 423 (2014); http://dx.doi.org/10.1016/j. anucene.2014.07.020.

2. G. PALMIOTTI and M. SALVATORES, "Use of Integral Experiments in the Assessment of Large Liquid-Metal Fast Breeder Reactor Basic Design Parameters," Nucl. Sci. Eng., 87, 333 (1984); http://dx.doi.org/10.13182/NSE87-333.

3. T. SANDA, F. NAKASHIMA, and K. SHIRAKATA, "Neutronic Decoupling and Space-Dependent Nuclear Characteristics for Large Liquid-Metal Fast Breeder Reactor Cores," Nucl. Sci. Eng., 113, 97 (1993); http://dx.doi. org/10.13182/NSE113-97.

4. G. RIMPAULT et al., "Flux Harmonics in Large SFR Cores in Relation with Core Characteristics such as Power Peaks," Proc. Int. Conf. Nuclear Mathematical and Computational Sciences (M\&C2013), Sun Valley, Idaho, May 5-9, 2013, American Nuclear Society (2013).

5. J. A. MAHAFFEY and M. V. DAVIS, "An Experimentally Measured Relationship Between Flux Tilt and Excess Reactivity," Ann. Nucl. Energy, 7, 489 (1980); http://dx.doi.org/ 10.1016/0306-4549(80)90094-8.

6. K. NISHINA and M. TOKASHIKI, "Verification of a More General Correspondence Between Eigenvalue Separation and Coupling Coefficient," Prog. Nucl. Energy, 30, 3, 277 (1996); http://dx.doi.org/10.1016/0149-1970(95)00089-5.

7. M. ANDOH et al., "Measurement of Flux Tilt and Eigenvalue Separation in Axially Decoupled Core," J. Nucl. Sci. Technol., 34, 5, 445 (1997); http://dx.doi.org/10.3327/jnst.34.445.

8. K. ISHITANI et al., "Measurement of Eigenvalue Separation by Using Position Sensitive Proportional Counter," Ann. Nucl. Energy, 25, 10, 721 (1998); http://dx.doi.org/10. 1016/S0306-4549(97)00042-X.

9. Y. KATO et al., "Analysis of First-Harmonic Eigenvalue Separation Experiments on KUCA Coupled Core," J. Nucl. Sci. Technol., 35, 3, 216 (1998); http://dx.doi.org/10.1080/ 18811248.1998.9733847.

10. C. H. PYEON et al., "Relationship Between Flux Tilt in Two-Energy-Group and Eigenvalue Separation," Ann. Nucl. Energy, 28, 1625 (2001); http://dx.doi.org/10.1016/ S0306-4549(01)00006-8.

11. C. H. PYEON et al., "Experiments and Analyses for Relationship Between Flux Tilt in Two-Energy-Group Model and First-Mode Eigenvalue Separation," J. Nucl. Sci. Technol., 41, 2, 171 (2004); http://dx.doi.org/10.1080/18811248. 2004.9715473. 
12. G. PALMIOTTI, "The 'FRENCH' (Flux Reconstructed Explicitly by Neutronic Calculated Harmonics) Method in Multidimensional and Multigroup Theory," Ann. Nucl. Energy, 14, 4, 167 (1987); http://dx.doi.org/10.1016/ 0306-4549(87)90014-4.

13. T. YAMAMOTO et al., "Effect of Higher-Harmonic Flux in Exponential Experiment for Subcriticality Measurement," J. Nucl. Sci. Technol., 40, 2, 77 (2003); http://dx. doi.org/10.1080/18811248.2003.9715336.

14. F. ZINZANI, C. DEMAZIERE, and C. SUNDE, "Calculation of the Eigenfunctions of the Two-Group Neutron Diffusion Equations and Application to Modal Decomposition of BWR Instabilities," Ann. Nucl. Energy, 35, 2109 (2008); http://dx.doi.org/10.1016/j.anucene.2008.05.004.

15. C. WANG et al., "On-Line Reconstruction of In-Core Power Distribution by Harmonics Expansion Methods," Nucl. Eng. Des., 241, 2, 3042 (2011); http://dx.doi.org/10. 1016/j.nucengdes.2011.05.013.

16. R. E. ALCOUFFE, "Diffusion Synthetic Acceleration Methods for the Diamond-Differenced Discrete Ordinates Equations," Nucl. Sci. Eng., 64, 344 (1977); http://dx.doi. org/10.13182/NSE77-1.

17. T. E. BOOTH, "Computing the Higher $k$-Eigenfunctions by Monte Carlo Power Iteration: A Conjecture," Nucl. Sci. Eng., 143, 291 (2003); http://dx.doi.org/10.13182/NSE02$10 \mathrm{TN}$.

18. T. E. BOOTH, "Power Iteration Method for the Several Largest Eigenvalues and Eigenfunctions," Nucl. Sci. Eng., 154, 48 (2006); http://dx.doi.org/10.13182/NSE05-05.

19. T. E. BOOTH and J. E. GUBERNATIS, "Exact Regional Monte Carlo Weight Cancellation for Second Eigenfunction Calculations," Nucl. Sci. Eng., 165, 283 (2010); http:// dx.doi.org/10.13182/NSE09-62.

20. T. YAMAMOTO, "Convergence of the Second Eigenfunction in Monte Carlo Power Iteration," Ann. Nucl. Energy, 36, 7 (2009); http://dx.doi.org/10.1016/j.anucene.2008.11. 004.

21. T. YAMAMOTO, "Higher Order $\alpha$ Mode Eigenvalue Calculation by Monte Carlo Power Iteration," Prog. Nucl. Sci. Technol., 2, 826 (2011); http://dx.doi.org/10.15669/pnst.2. 826.

22. B. SHI and B. PETROVIC, "Analysis of the Higher Eigenfunction Calculation Using a Modified Power Iteration Method," Proc. Int. Conf. Advances in Reactor Physics to Power the Nuclear Renaissance (PHYSOR 2010),
Pittsburgh, Pennsylvania, May 9-14, 2010, American Nuclear Society (2010).

23. B. SHI and B. PETROVIC, "Calculating the Second Eigenpair in Criticality Calculations Using the Monte Carlo Method with Source Points Pairing as an Efficient NetWeight (Cancellation) Algorithm," Nucl. Sci. Eng., 172, 138 (2012); http://dx.doi.org/10.13182/NSE11-19.

24. J. S. WARSA et al., "Krylov Subspace Iterations for Deterministic k-Eigenvalue Calculations," Nucl. Sci. Eng., 147, 26 (2004); http://dx.doi.org/10.13182/NSE04-1.

25. R. S. MODAK and A. GUPTA, "New Applications of Orthomin(1) Algorithm for $K$-Eigenvalue Problem in Reactor Physics," Ann. Nucl. Energy, 33, 538 (2006); http://dx. doi.org/10.1016/j.anucene.2005.10.003.

26. R. S. MODAK and V. K. JAIN, "Sub-Space Iteration Scheme for the Evaluation of $\lambda$-Modes of FiniteDifferenced Multigroup Neutron Diffusion Equations," Ann. Nucl. Energy, 23, 3, 229 (1996); http://dx.doi.org/10. 1016/0306-4549(95)00015-6.

27. T. KITADA and T. TAKEDA, "Evaluation of Eigenvalue Separation by the Monte Carlo Method," J. Nucl. Sci. Technol., 39, 2, 129 (2002); http://dx.doi.org/10.1080/18811248. 2002.9715166

28. M. GOLDSMITH, "Symmetry Properties of Some Eigenfunctions Occurring in Reactor Analysis," Nucl. Sci. Eng., 17, 111 (1963); http://dx.doi.org/10.13182/NSE17-111-124.

29. M. MAKAI, "Symmetries Applied to Reactor Calculations," Nucl. Sci. Eng., 82, 338 (1982); http://dx.doi.org/10. 13182/NSE82-338.

30. R. S. MODAK and A. GUPTA, "Use of a New Boundary Condition in Computational Neutron Transport," Nucl. Sci. Eng., 163, 263 (2009); http://dx.doi.org/10.13182/NSE163263.

31. G. B. BRUNA and A. SARGENI, "A Computational Technique for Evaluating Eigenfunctions of Symmetrical Nuclear Systems," Ann. Nucl. Energy, 21, 12, 745 (1994); http://dx.doi.org/10.1016/0306-4549(94)90023-X.

32. M. MAILLOT, J. TOMMASI, and G. RIMPAULT, "Calculation of Higher-Order Fluxes in Symmetric Cores-II: Implementation," Nucl. Sci. Eng., 184, 190 (2016); http:// dx.doi.org/10.13182/NSE16-5.

33. B. J. McCARTIN, Laplacian Eigenstructure of the Equilateral Triangle, Hikari Ltd (2011). 